\title{
Chaos Control in Fractional Order Smart Grid with Adaptive Sliding Mode Control and Genetically Optimized PID Control and Its FPGA Implementation
}

\author{
Anitha Karthikeyan and Karthikeyan Rajagopal \\ Centre for Non-Linear Dynamics, Defense University, Bishoftu, Ethiopia \\ Correspondence should be addressed to Karthikeyan Rajagopal; rkarthiekeyan@gmail.com
}

Received 16 January 2017; Revised 1 March 2017; Accepted 9 March 2017; Published 13 April 2017

Academic Editor: Viet-Thanh Pham

Copyright (C) 2017 Anitha Karthikeyan and Karthikeyan Rajagopal. This is an open access article distributed under the Creative Commons Attribution License, which permits unrestricted use, distribution, and reproduction in any medium, provided the original work is properly cited.

\begin{abstract}
We investigate a specific smart grid system and its nonlinear properties. Lyapunov exponents are derived to prove the existence of chaos and bifurcation and bicoherence contours are investigated to show the parameter dependence and existence of quadratic nonlinearities, respectively. A fractional order model of the smart grid system (FOSG) is then derived and bifurcation of the FOSG system with variation in the commensurate fractional order of the system is investigated to show that largest Lyapunov exponent of the system exists in fractional order. Hence we proposed two different control methods to suppress the chaotic oscillations. In the first method we derive fractional order adaptive sliding mode control (FOASMC) algorithm to control chaotic oscillations and in the second method we used genetically optimized fractional order PID controllers (GAFOPID) for chaos control. Numerical simulations are conducted to show the effectiveness of the controllers and also to prove that GAFOPID controllers are more effective than FOASMC controllers for fractional order systems. The GAFOPID controllers are then realized in FPGA to show that the proposed methodology is hardware realizable.
\end{abstract}

\section{Introduction}

Smart grid holds the promise that the power sector can go "green" by taking more aggressive measures to lower the carbon dioxide $\left(\mathrm{CO}_{2}\right)$ emissions through efficient integration of renewable energy sources $[1,2]$. Smart grids and its technologies modernize our electric grids ensure safe, secure, cost effective, and reliable power transmission [3,4]. Nonlinear phenomenon in power systems such as voltage collapse and oscillatory phenomenon can be analyzed using chaos theory [5]. Unpredictability and high sensitivity to initial conditions are the important characters of chaotic systems which led to severe blackouts and power system failures [6].

Control of chaotic systems in science and engineering is an important research area in the control literature [7]. Particularly chaos control methodologies have been of greater use in nonlinearity suppression of electrical systems such as in brushless DC motor [8], bifurcation and chaos control in a permanent magnet synchronous motor $[9,10]$, and PI controlled induction motor [11]. Uncertainty plays an important role in controlling nonlinearity in power systems. As discussed in literatures, the uncertainty caused by the state changes because of parallel distribution and generation in smart grids are not investigated $[12,13]$.

Recently many researchers have shown interest in the study of fractional order nonlinear systems [14-16]. Methods for simulating fractional order chaotic system were presented by Petras [17] and Matlab solutions for numerical analysis of fractional order chaotic systems were discussed by Trzaska [18]. In chaos control of electrical systems fractional order controllers play an important role as larger Lyapunov exponents occur when fractional orders are close to "1" [1921]. Chaos suppression in fractional order brushless DC motor using sliding mode controllers [19] and fractional order permanent magnet synchronous motor, pi controlled induction motor by extended back stepping control [20], and fractional order PMSM motors with adaptive nonlinear controllers [21] is earlier investigated in the literatures. The 
stability of fractional order systems using Lyapunov stability theory has been investigated in the literature [22, 23]. A fractional order controller to stabilize the unstable fixed points of an unstable open-loop system was proposed by Tavazoei and Haeri [24]. A delayed feedback control (DFC) based on the act-and-wait concept for nonlinear dynamical systems was proposed by Konishi et al. [25], which reduces the dynamics of DFC systems to that of discrete-time systems. The control and synchronization of an induction motor system were investigated by Jin et al. [26]. Fractional order controller design for brushless DC motors and induction motors were investigated in the literature [19, 20]. FPGA implementation of fractional order systems is first discussed by Rajagopal et al. [27, 28].

Recently many digital implementations of chaotic systems have been proposed and implemented in field programmable gate arrays (FPGA) [29-31]. Image crypto systems using chaotic random number generators are implemented in FPGA and are of greater interest because of the robustness and complexity of the chaos encryption systems [32]. FPGA based weak signal detectors using a Duffing oscillator were proposed by Rashtchi and Nourazar [33]. Multiscroll attractors and their digital implementation using FPGA are extensively investigated in recent years [29, 34]. Memristor based chaotic system and its FPGA circuits were proposed by Xu et al. [35].

Motivated by the above discussions, in this paper we propose a chaos control scheme for a fractional order smart grid model with fractional order adaptive sliding mode controllers (FOASMC) and genetically optimized fractional order PID controllers (FOGAPID). Dynamic analyses of the specific smart grid integer order dimensionless model [36] like Lyapunov exponents and bifurcation and bicoherence plots are derived to prove that chaos exists for selective values of the parameters. The fractional order smart grid (FOSG) model is then derived from the integer order smart grid model [36]. Bifurcation analysis of the FOSG system for variations fractional orders is investigated to prove that the largest Lyapunov exponent exists when fractional order is close to "1." Hence fractional order adaptive sliding mode (FOASM) controllers and genetically optimized fractional order PID controllers are proposed to suppress the chaotic oscillations. By numerical simulations GAFOPID controllers are proven effective compared to the FOASM controllers. The proposed GAFOPID controllers are then implemented in FPGA to show that the proposed control scheme is hardware realizable.

\section{Chaotic Dynamics of Smart Grid}

In this section we investigate the chaotic dynamics of the specific smart grid system [36]. The dynamics of the system depends on rotator angle $\delta_{t}$, load voltage angle $\delta_{L}$, load voltage $V_{L}$, angular rotation $\omega$, and inverter current $i_{0}$. To analyze the chaotic behavior of the smart grid parameters $\left[\delta_{t}, \omega, \delta_{L}, V_{L}\right]^{T}$ are taken as fixed parameters and $i_{0}$ is taken as the variable parameter. For simplicity the parameters are assumed as $x=\left[x_{1}, x_{2}, x_{3}, x_{4}\right]^{T}=\left[\delta_{t}, \omega, \delta_{L}, V_{L}\right]^{T}$. The dimensionless model of the smart grid system is as given in

$$
\begin{aligned}
\dot{x}_{1}= & x_{2}, \\
\dot{x}_{2}= & 0.573-0.167 x_{2}+20 x_{4} \cos \left(x_{1}-x_{3}+1.483\right) \\
& +11.667 x_{4} \cos \left(x_{1}+1.483\right), \\
\dot{x}_{3}= & 69-93.33 x_{4}-179.05 x_{4}^{2}-50 i_{0} x_{4} \sin x_{3} \\
& -300 x_{4} \sin x_{3} \cos \left(x_{1}-1.483\right), \\
\dot{x}_{4}= & 25.322 x_{4}^{2}+13.054 x_{4}+3.529 x_{4} \cos \left(x_{1}-1.483\right) \\
& -3.529 x_{4} \cos x_{3} \cos \left(x_{3}-1.483\right) \\
& +42.353 x_{4} \sin x_{3} \cos \left(x_{1}-1.483\right) \\
& +7.059 i_{0} x_{4} \sin x_{3} \\
& -42.353 x_{4} \sin x_{3} \cos \left(x_{3}-1.483\right) \\
& -35.294 x_{4} \sin \left(x_{3}+1.483\right) \\
& +0.588 i_{0} x_{4} \cos x_{3}+2.941 x_{4} \cos \left(x_{3}-1.483\right) \\
& +1.31778 .
\end{aligned}
$$

System (1) shows chaotic behavior for the selected initial conditions $[0.3,0.2,0.1,0.97]$ and $i_{0}=0.01$. Figure 1 shows the $3 \mathrm{D}$ phase portrait of the smart grid system (1).

\section{Dynamic Analysis of the Smart Grid System}

In this section we investigate the properties of the smart grid system discussed in Section 2. The initial conditions for the analysis are taken as in Section 2.

3.1. Equilibrium Points and Jacobian Matrix. The smart grid system (1) has one unstable equilibrium point $E_{1}=$ $0.7336,0,0.8731,0.2278$. The characteristic equation of system (1) is given as $\lambda^{4}+32.4 \lambda^{3}+530.9 \lambda^{2}+444.29 \lambda-4541$ and the eigenvalues are $\lambda_{1,2}=-15.46 \pm 15.98 i, \lambda_{3}=-3.85, \lambda_{4}=2.38$ and $\lambda_{4}$ is the unstable focus.

The Jacobian matrix of the smart grid system (1) at any point $X \in R^{4}$ is found as

$$
J(X)=\left[\begin{array}{cccc}
0 & 1 & 0 & 0 \\
f_{5}(x) & 0.167 & f_{9}(x) & f_{6}(x) \\
f_{10}(x) & 0 & f_{8}(x) & f_{3}(x) \\
f_{4}(x) & 0 & f_{1}(x) & f_{2}(x)
\end{array}\right]
$$

and the elements of matrix are the functions defined as

$$
\begin{aligned}
f_{1}(x)= & 3.529 x_{4} \sin x_{3} \cos \left(x_{3}-1.483\right) \\
& +3.529 x_{4} \cos x_{3} \sin \left(x_{3}-1.483\right)
\end{aligned}
$$




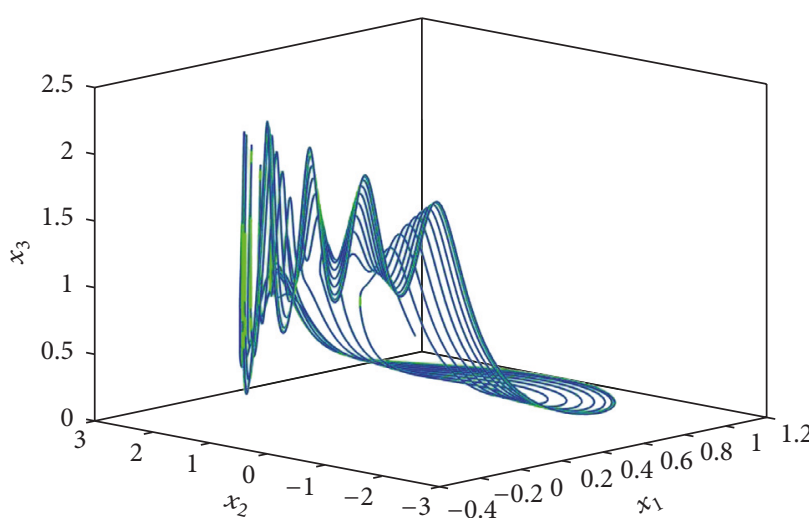

(a)

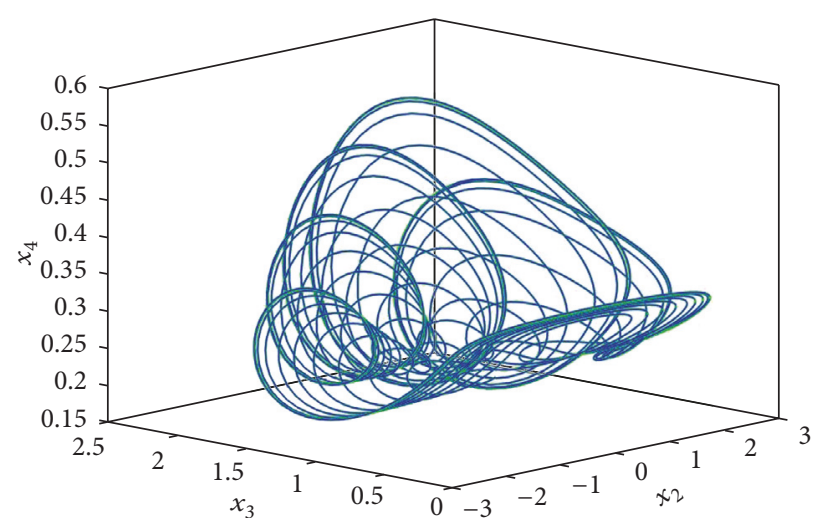

(b)

Figure 1: 3D phase portraits of the smart grid system (1).

$$
\begin{aligned}
& +42.353 x_{4} \cos x_{3} \cos \left(x_{1}-1.483\right) \\
& +7.059 i_{0} x_{4} \cos x_{3} \\
& -42.353 x_{4} \cos x_{3} \cos \left(x_{3}-1.483\right) \\
& +42.353 x_{4} \sin x_{3} \sin \left(x_{3}-1.483\right) \\
& -35.294 x_{4} \cos \left(x_{3}+1.483\right) \\
& -0.588 i_{0} x_{4} \sin x_{3} \\
& -2.941 x_{4} \sin \left(x_{3}-1.483\right), \\
f_{2}(x)= & 50.644 x_{4}+13.054+3.529 \cos \left(x_{1}-1.483\right) \\
& -3.529 \cos x_{3} \cos \left(x_{3}-1.483\right) \\
& +42.353 \sin x_{3} \cos \left(x_{1}-1.483\right) \\
& +7.059 i_{0} \sin x_{3} \\
& -42.353 \sin x_{3} \cos \left(x_{3}-1.483\right) \\
& -35.294 \sin \left(x_{3}+1.483\right)+0.588 i_{0} \cos x_{3} \\
& +2.941 \cos \left(x_{3}-1.483\right), \\
f_{5}(x)= & -20 x_{4} \sin \left(x_{1}-x_{3}+1.483\right) \\
& -11.667 x_{4} \sin \left(x_{1}+1.483\right), \\
f_{4}(x)= & -3.529 x_{4} \sin \left(x_{1}-1.483\right) \\
& +11.667 \cos \left(x_{1}-x_{3}+1.483\right) \\
f_{3}(x)= & -93.33-358.1 x_{4}-50 i_{0} \sin x_{3} \\
& -300 \sin x_{3} \cos \left(x_{1}-1.483\right), \\
& 42.353 x_{4} \sin x_{3} \sin \left(x_{1}-1.483\right), \\
&
\end{aligned}
$$

$$
\begin{aligned}
f_{7}(x)= & -50 i_{0} x_{4} \cos x_{3} \\
& +300 x_{4} \cos x_{3} \cos \left(x_{1}-1.483\right), \\
f_{9}(x)= & 20 x_{4} \sin \left(x_{1}-x_{3}+1.483\right), \\
f_{10}(x)= & 300 x_{4} \sin x_{3} \sin \left(x_{1}-1.483\right) .
\end{aligned}
$$

3.2. Lyapunov Exponents and Kaplan-Yorke Dimension. Lyapunov exponents of a nonlinear system define the convergence and divergence of the states. The existence of positive Lyapunov exponents confirms the chaotic behavior of the system $[37,38]$. We take the parameters of the smart grid system (1) with initial conditions and parameters as in Section 2; then the Lyapunov exponents of the smart grid system (1) are numerically found as

$$
\begin{aligned}
& L_{1}=0.270809, \\
& L_{2}=-1.408465, \\
& L_{3}=-7.882472, \\
& L_{4}=-25.888492 .
\end{aligned}
$$

Since there are positive Lyapunov exponents in (4), it is clear that the smart grid system (1) is chaotic. Figure 2 shows the Lyapunov exponents of the smart grid system. We note that the sum of the Lyapunov exponents of system (1) is negative. In fact,

$$
L_{1}+L_{2}+L_{3}+L_{4}=-34.908<0 .
$$

This shows that the smart grid system (1) is dissipative.

Also, the Kaplan-Yorke dimension of the smart grid system (1) is derived as

$$
D_{\mathrm{KY}}=3+\frac{L_{1}+L_{2}+L_{3}}{L_{4}}=3.3484 .
$$

3.3. Bifurcation. In order to understand the parameter dependence of the smart grid system, we fix all the other 


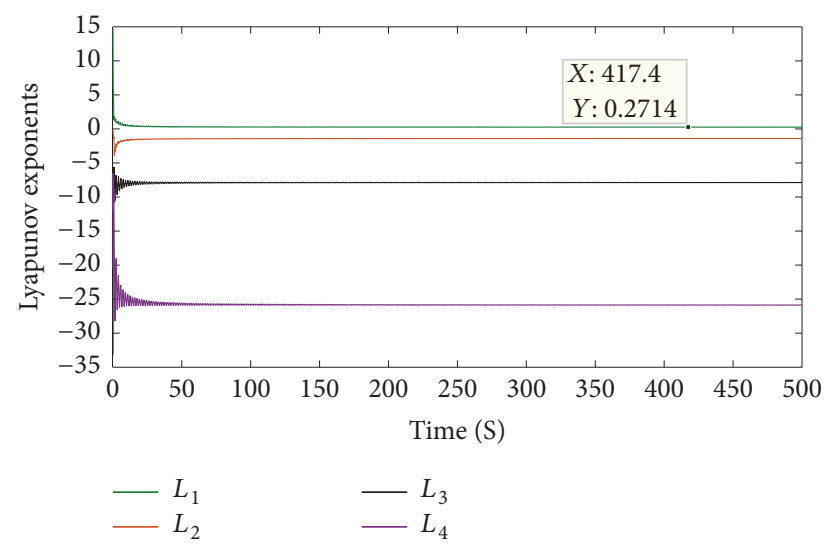

FIGURE 2: Lyapunov exponents of the smart grid system (1).

parameters $\left(\left[x_{1}, x_{2}, x_{3}, x_{4}\right]^{T}=\left[\delta_{t}, \omega, \delta_{L}, V_{L}\right]^{T}\right), i_{0}$ is varied, and the behavior of the integer order smart grid system (1) is investigated. The bifurcation plot for various states versus the inverter current $i_{0}$ is given by Figures 3(a), 3(b), 4(a), and 4(b). From the figures, it is clear that, for the values $0 \leq i_{0} \leq 2.78$, the system shows denser points confirming the existence of system's largest positive Lyapunov exponent. Therefore, from the Lyapunov exponents and bifurcation diagrams in these figures, a conclusion can be obtained that chaos exists in smart grid system (1) for a certain range of parameters.

3.4. Bicoherence. The bicoherence or the normalized bispectrum is a measure of the amount of phase coupling that occurs in a signal or between two signals. Both bicoherence and bispectrum are used to find the influence of a nonlinear system on the joint probability distribution of the system input. Phase coupling is the estimate of the proportion of energy in every possible pair of frequency components $\left(f_{j}, f_{k}\right)$. Bicoherence analysis is able to detect coherent signals in extremely noisy data, provided that the coherency remains constant for sufficiently long times, since the noise contribution falls off rapidly with increasing number of segments $N$.

The autobispectrum of a chaotic system is given by Pezeshki [39]. He derived the autobispectrum with the Fourier coefficients

$$
B\left(\omega_{1}, \omega_{2}\right)=E\left[A\left(\omega_{1}\right) A\left(\omega_{2}\right) A^{*}\left(\omega_{1}+\omega_{2}\right)\right],
$$

where $\omega_{n}$ is the radian frequency and $A$ is the Fourier coefficients of the time series. The normalized magnitude spectrum of the bispectrum known as the squared bicoherence is given by

$$
b\left(\omega_{1}, \omega_{2}\right)=\frac{\left|B\left(\omega_{1}, \omega_{2}\right)\right|^{2}}{P\left(\omega_{1}\right) P\left(\omega_{2}\right) P\left(\omega_{1}+\omega_{2}\right)},
$$

where $P\left(\omega_{1}\right)$ and $P\left(\omega_{2}\right)$ are the power spectrums at $f_{1}$ and $f_{2}$.

The motivation to study the bicoherence is twofold. First, the bicoherence can be used to extract information due to deviations from Gaussianity and suppress additive (colored) Gaussian noise. Second, the bicoherence can be used to detect and characterize asymmetric nonlinearity in signals via quadratic phase coupling or identify systems with quadratic nonlinearity. The bicoherence is the third-order spectrum. Whereas the power spectrum is a second-order statistics, formed from $X^{\prime}(f) * X(f)$, where $X(f)$ is the Fourier transform of $x(t)$, the bispectrum is third-order statistics formed from $X\left(f_{j}\right) * X\left(f_{k}\right) * X^{\prime}\left(f_{j}+f_{k}\right)$. The bispectrum is therefore a function of a pair of frequencies $\left(f_{j}, f_{k}\right)$. It is also a complexvalued function. The (normalized) square amplitude is called the bicoherence (by analogy with the coherence from the cross-spectrum). The bispectrum is calculated by dividing the time series into $M$ segments of length $N$ _seg, calculating their Fourier transforms and biperiodogram, then averaging over the ensemble. Although the bicoherence is a function of two frequencies the default output of this function is a one-dimensional output, with the bicoherence refined as a function of only the sum of the two frequencies.

Figures 5 and 6 depict the contours of the bicoherence of the states $x$ and $y$ for the smart grid system (1). Figure 7 shows the bicoherence contours of all the states together. Shades in yellow represent the multifrequency components contributing to the power spectrum. From Figures 5, 6, and 7 , the cross-bicoherence is significantly nonzero, and nonconstant, indicating a nonlinear relationship between the states. In Figures 5 and 6 (autobicoherence) the yellow shades indicate that the nonlinear relationship of the states $x$ and $y$ is not narrowband processes. The yellow shades and nonsharpness of the peaks, as well as the presence of structure around the origin in Figure 7 (cross-bicoherence), indicate that the nonlinearity between the states $x, y, z, w$ is not of the quadratic nonlinearity and hence may be because of nonlinearity of higher dimensions. The most two dominant frequencies $\left(f_{1}, f_{2}\right)$ are taken for deriving the contour of bicoherence. The sampling frequency $\left(f_{s}\right)$ is taken as the reference frequency. Direct FFT is used to derive the power spectrum for individual frequencies and Hankel operator is used as the frequency mask. Hanning window is used as the FIR filter to separate the frequencies.

\section{Fractional Order Current Driven Induction Motor}

In this section we will derive the dimensionless fractional order model of the induction motor. The dimensionless integer order model of a PI speed regulated current driven induction motor is given by [36] as follows:

$$
\begin{aligned}
D^{q_{1}} x_{1}= & x_{2} \\
D^{q_{2}} x_{2}= & 0.573-0.167 x_{2}+20 x_{4} \cos \left(x_{1}-x_{3}+1.483\right) \\
& +11.667 x_{4} \cos \left(x_{1}+1.483\right) \\
D^{q_{3}} x_{3}= & 69-93.33 x_{4}-179.05 x_{4}^{2}-50 i_{0} x_{4} \sin x_{3} \\
& -300 x_{4} \sin x_{3} \cos \left(x_{1}-1.483\right) \\
D^{q_{4}} x_{4}= & 25.322 x_{4}^{2}+13.054 x_{4} \\
& +3.529 x_{4} \cos \left(x_{1}-1.483\right)
\end{aligned}
$$




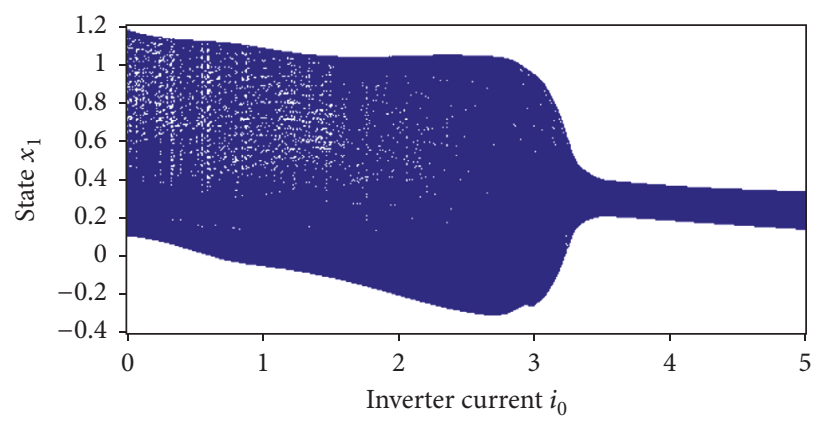

(a) Bifurcation of state $x_{1}$ with $i_{0}$

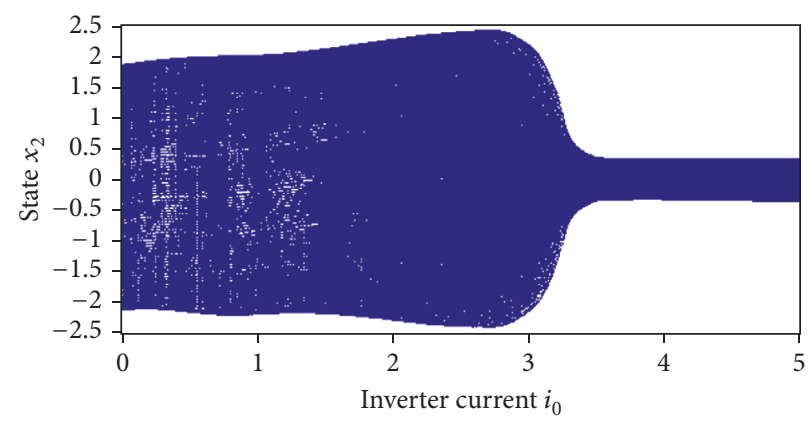

(b) Bifurcation of state $x_{2}$ with $i_{0}$

Figure 3

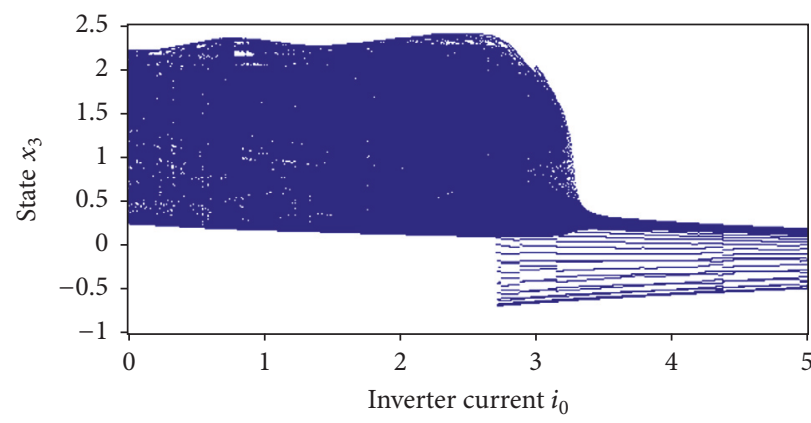

(a) Bifurcation of state $x_{3}$ with $i_{0}$

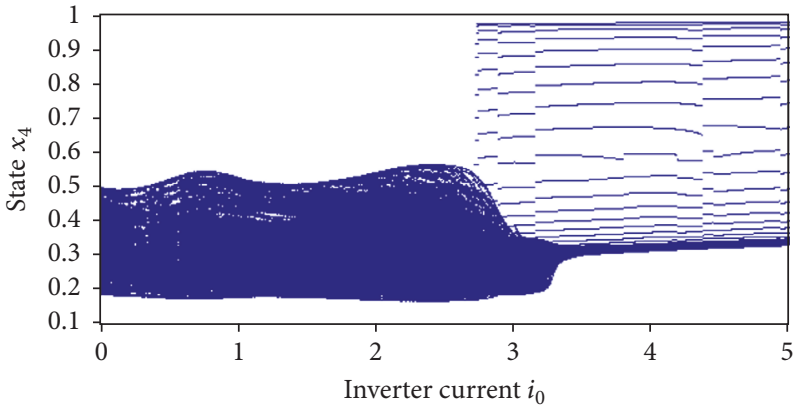

(b) Bifurcation of state $x_{4}$ with $i_{0}$

Figure 4
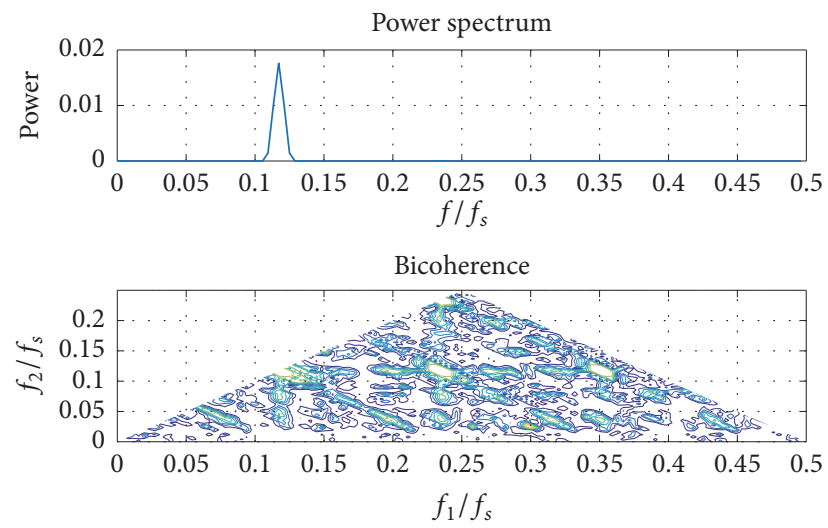

Figure 5: Contour of bicoherence of state $x$ for the smart grid system.
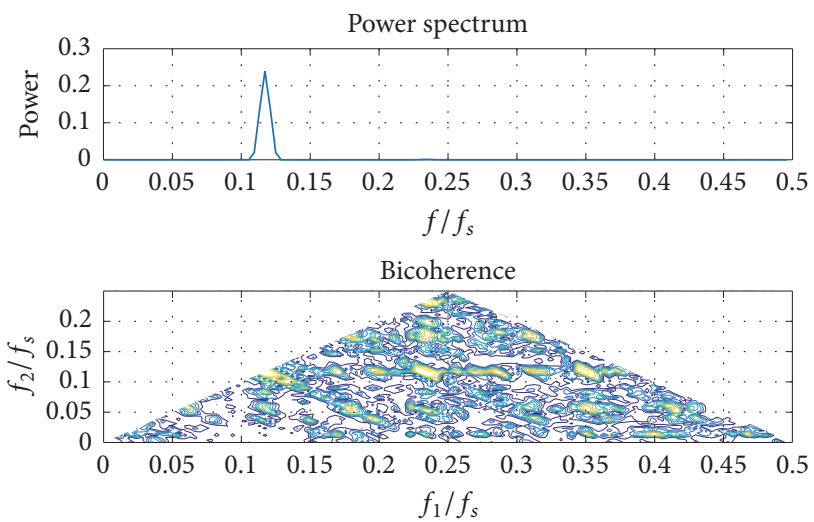

FIGURE 6: Contour of bicoherence of state $y$ for the smart grid system.

$$
\begin{aligned}
& +0.588 i_{0} x_{4} \cos x_{3} \\
& +2.941 x_{4} \cos \left(x_{3}-1.483\right)+1.31778
\end{aligned}
$$

The initial conditions and the parameters are the same as discussed for the integer order model in Section 2. The fractional orders $q_{i}$ with $i=1,2,3,4$ are kept close to " 1 " to get 

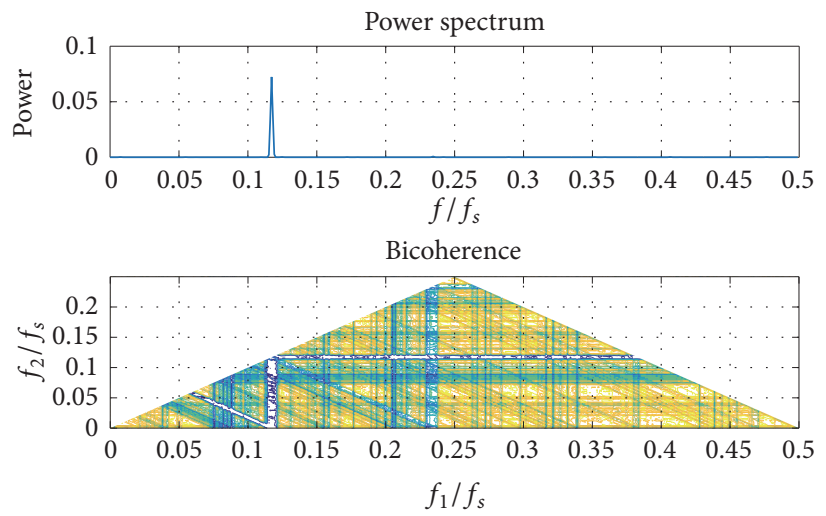

FIGURE 7: Contour of bicoherence of all the states of the smart grid system.

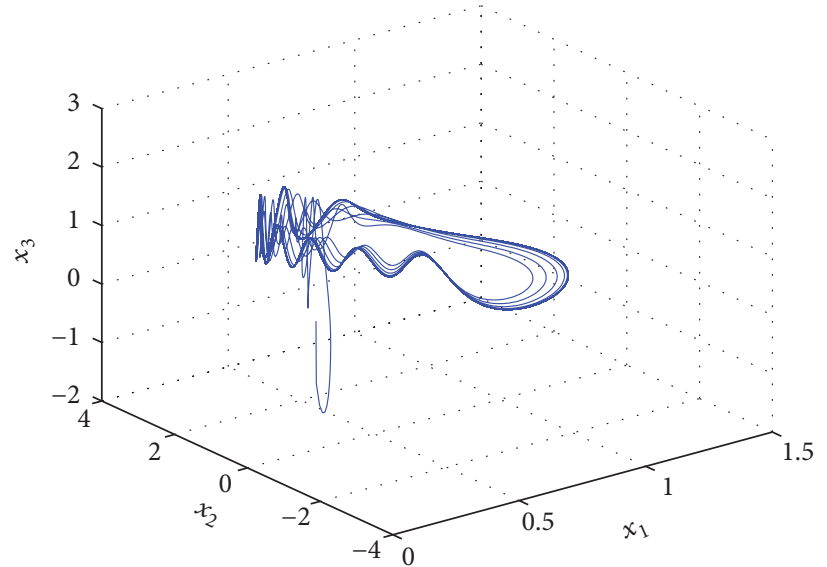

(a)

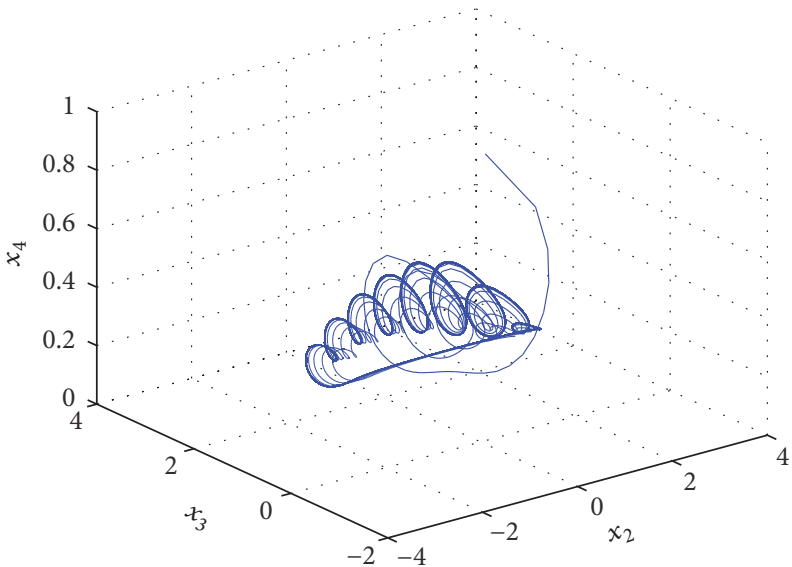

(b)

FiguRE 8: 3D phase portraits of the fractional order smart grid system.

the largest Lyapunov exponents. Figure 8 shows the 3D phase portraits of the fractional order smart grid (FOSG) system for $q_{i}=992$.

\section{Dynamic Analysis of the FOSG System}

5.1. Bifurcation with Fractional Order. Most of the dynamic properties of the integer order system (1) like the Lyapunov exponents and bifurcation with parameters are preserved in the fractional order if $q_{i}>0.985$, where $i=1,2,3,4$. The most important analysis of interest when investigating a fractional order system is the bifurcation with fractional orders. Figure 9(a) shows the bifurcation of the FOSG system with commensurate fractional order $q_{1}=q_{2}=q_{3}=q_{4}=q$ and Figure 9 (b) shows the 3D state portrait $\left(x_{2}, x_{3}, x_{4}\right.$ plane $)$ of the FOSG system for various commensurate fractional orders $q_{1}=q_{2}=q_{3}=q_{4}=q$. The largest positive Lyapunov exponent $\left(L_{1}=0.271921\right)$ of the FOSG system appears when $q=0.992$ against its largest integer order Lyapunov exponent $\left(L_{1}=0.270809\right)$. Hence fractional order chaos suppression/control proves efficient compared to the integer order controls as the systems show the largest positive
Lyapunov exponent in fractional order close to "1." It can also be seen that, as the fractional order $q$ decreases, the FOSG system starts losing its largest positive Lyapunov exponent. When $q \leq 0.98$ the only positive Lyapunov exponent of the system becomes negative and thus the chaotic oscillations in the system disappear.

\subsection{Stability Analysis of FOSG System}

Commensurate Order. For commensurate FOSG system of order $q$, the system is stable and exhibits chaotic oscillations if $\left|\arg \left(\operatorname{eig}\left(J_{E}\right)\right)\right|=\left|\arg \left(\lambda_{i}\right)\right|>q \pi / 2$, where $J_{E}$ is the Jacobian matrix at the equilibrium $E$ and $\lambda_{i}$ are the eigenvalues of the FOSG system, where $i=1,2,3,4$. As seen from the FOSG system, the eigenvalues should remain in the unstable region and the necessary condition for the FOSG system to be stable is $q>(2 / \pi) \tan ^{-1}(|\operatorname{Im} \lambda| / \operatorname{Re} \lambda)$. As we know, the eigenvalues of the system are $\lambda_{1,2}=-15.46 \pm 15.98 i, \lambda_{3}=-3.85, \lambda_{4}=2.38$ and it is clearly seen that $\lambda_{4}$ is an unstable focus and hence $\lambda_{4}$ contributes to the existence of chaotic oscillations in FOSG system. 
Complexity

7
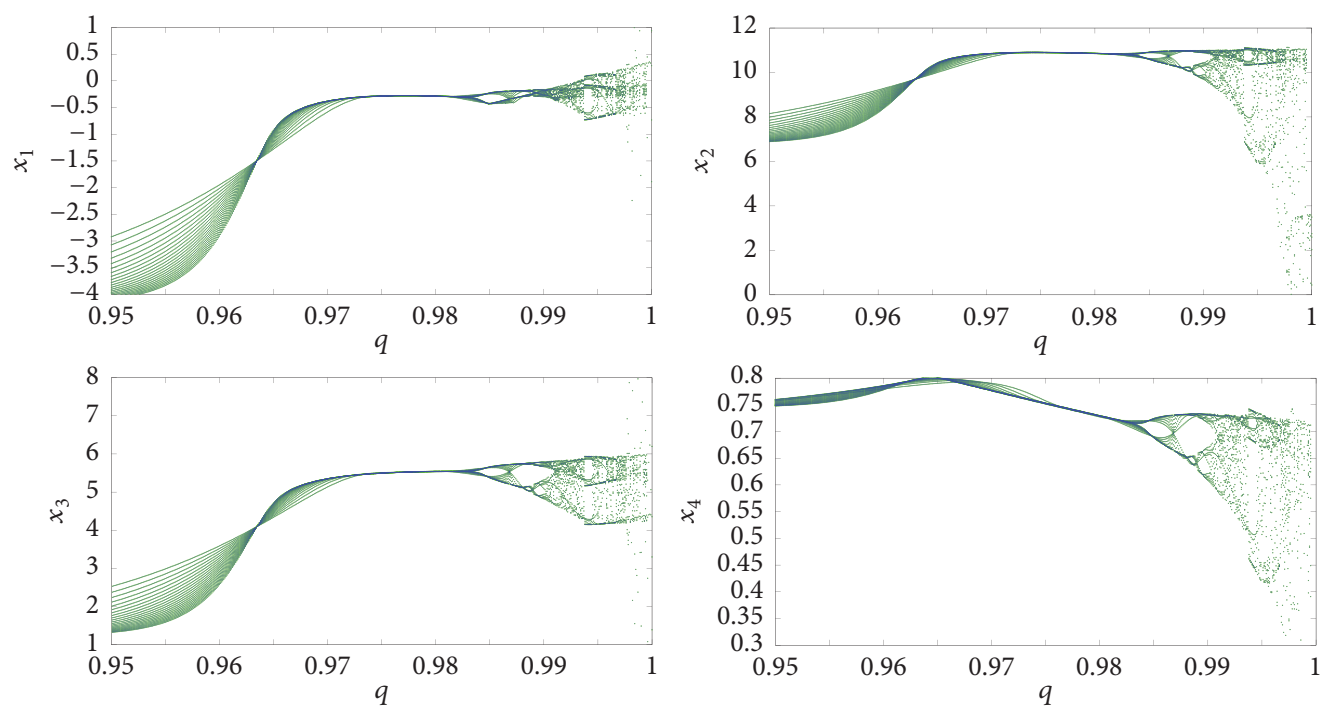

(a) Bifurcation with fractional orders for FPSG system

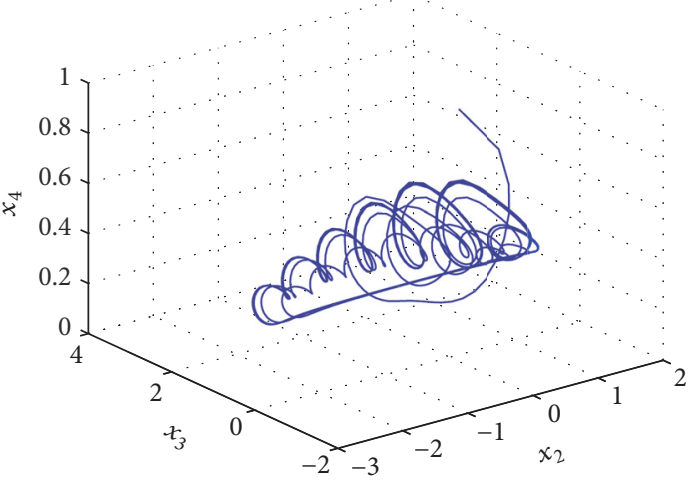

(A) $q=0.999 \& L_{1}=0.27090$

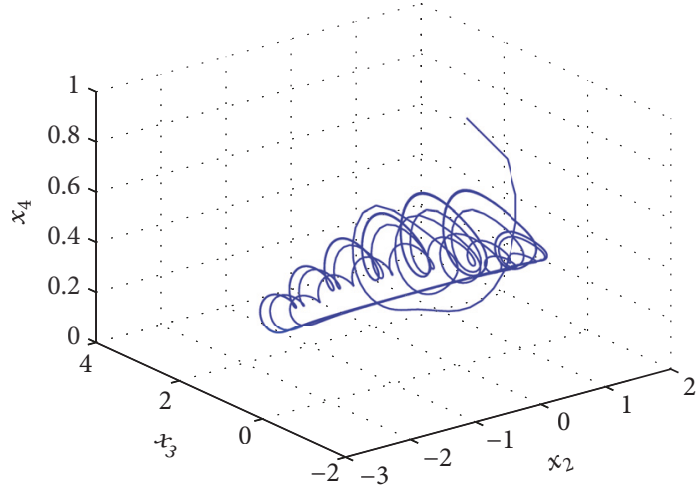

(B) $q=0.998 \& L_{1}=0.27093$

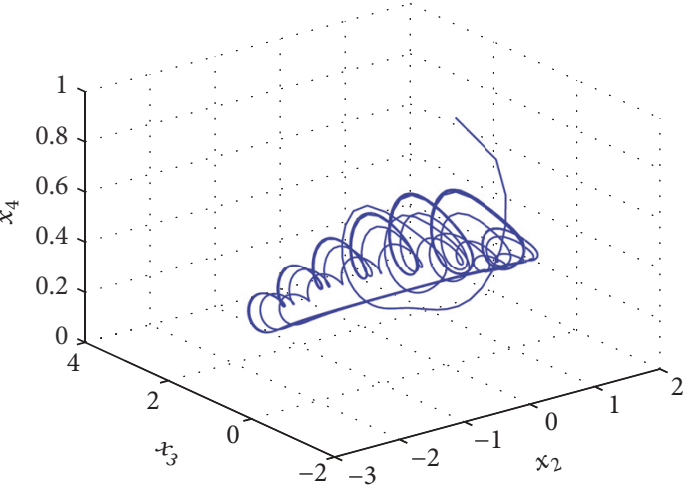

(C) $q=0.997 \& L_{1}=0.27102$

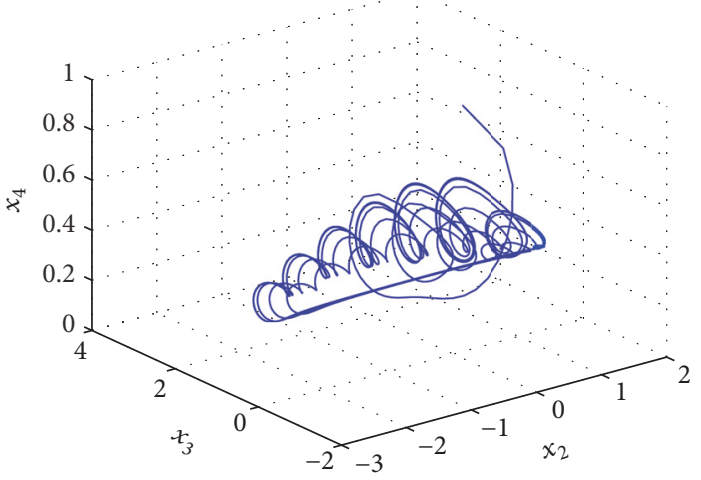

(E) $q=0.995 \& L_{1}=0.27124$

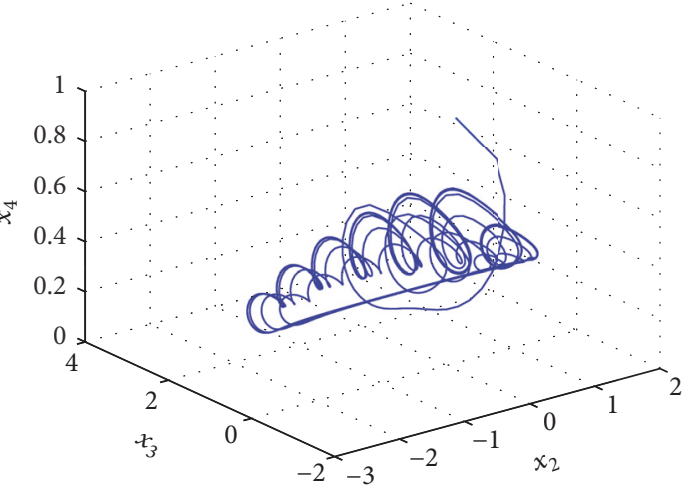

(D) $q=0.996 \& L_{1}=0.27110$

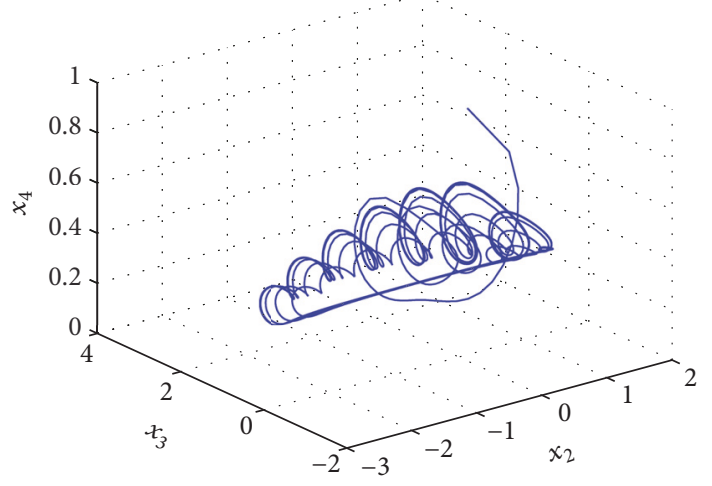

(F) $q=0.994 \& L_{1}=0.27147$

Figure 9: Continued. 


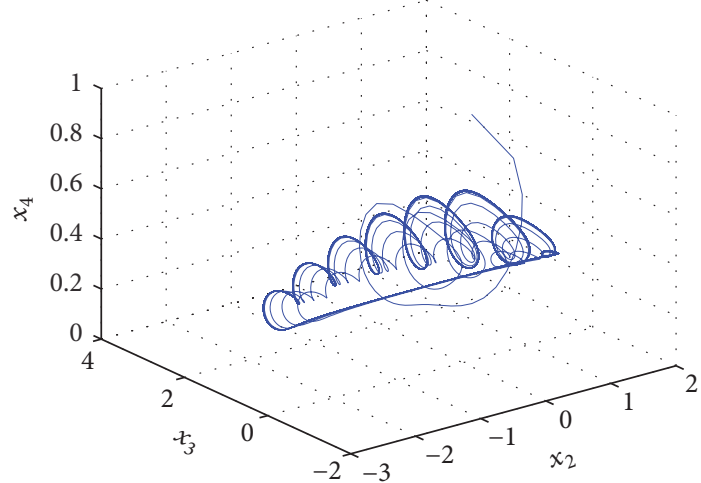

(G) $q=0.993 \& L_{1}=0.27165$

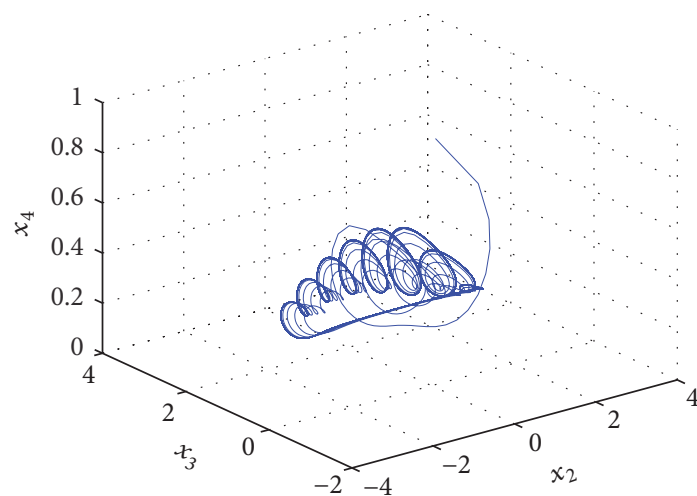

(I) $q=0.991 \& L_{1}=0.25158$

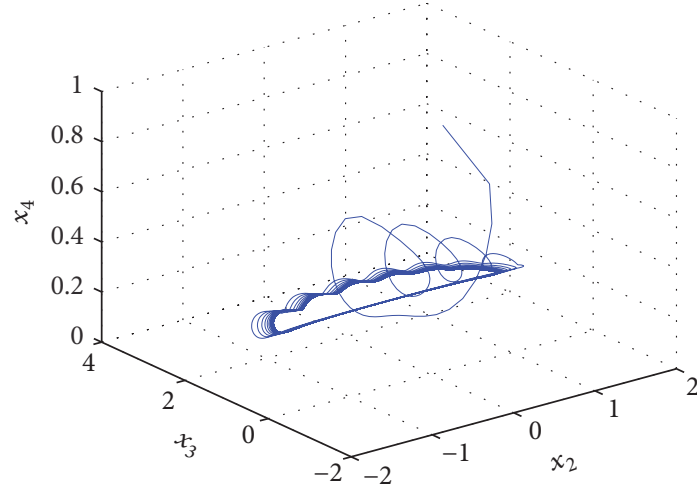

(K) $q=0.97 \& L_{1}=0.03170$

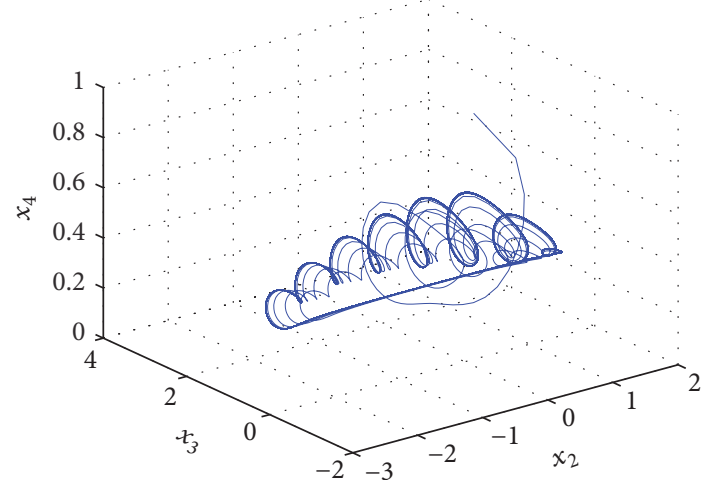

(H) $q=0.992 \& L_{1}=0.27192$

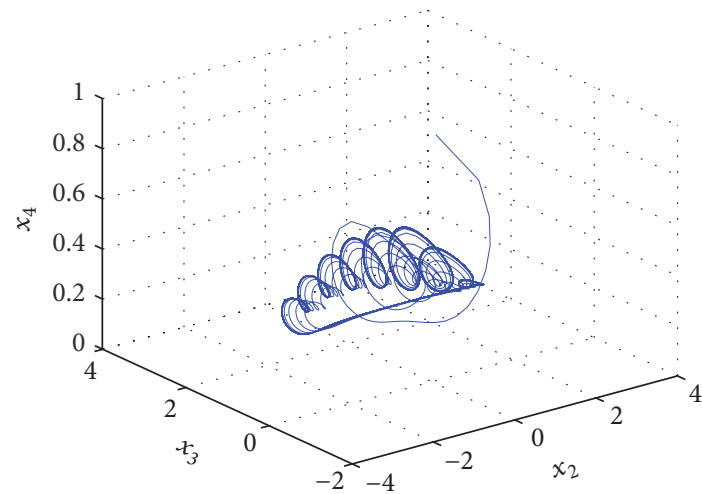

(J) $q=0.99 \& L_{1}=0.23011$

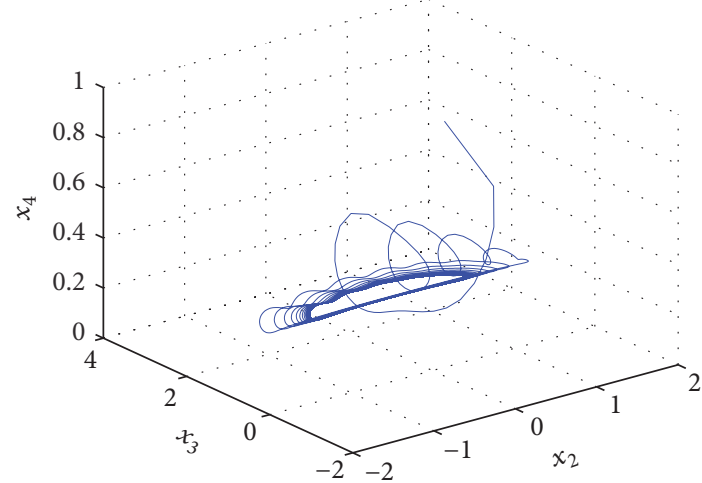

(L) $q=0.96 \& L_{1}=-0.10911$

(b) $3 \mathrm{D}$ phase portraits $\left(x_{2}, x_{3}, x_{4}\right)$ of the FOSG system for variations in fractional order

FiguRE 9

Incommensurate Order. The necessary condition for the FOSG system to exhibit chaotic oscillations in the incommensurate case is $\pi / 2 M-\min _{i}\left(\left|\arg \left(\lambda_{i}\right)\right|\right)>0$, where $M$ is the LCM of the fractional orders. If $q_{x}=0.99, q_{y}=0.98$, $q_{z}=0.97$, and $q_{w}=0.96$, then $M=100$. The characteristic equation of the system evaluated at the equilibrium is $\operatorname{det}\left(\operatorname{diag}\left[\lambda^{M q_{x}}, \lambda^{M q_{y}}, \lambda^{M q_{z}}, \lambda^{M q_{w}}\right]-J_{E}\right)=0$ and by substituting the values of $M$ and the fractional orders, $\operatorname{det}\left(\operatorname{diag}\left[\lambda^{99}, \lambda^{98}, \lambda^{97}, \lambda^{96}\right]-J_{E}\right)=0$, the characteristic equation for the equilibrium point $E_{1}$ is $\lambda^{390}+\lambda^{295}$ $+\lambda^{294}+33.21 \lambda^{293}+1.17 \lambda^{292}+\lambda^{199}+33.23 \lambda^{198}+$ $521.14 \lambda^{197}+33.40 \lambda^{196}+38.59 \lambda^{195}+0.167 \lambda^{194}+6.56 \lambda^{193}$ $+\lambda^{102}+33.40 \lambda^{101}+557.57 \lambda^{100}+606.81 \lambda^{99}+38.94 \lambda^{98}-$ $0.18 \lambda^{97} 369.75 \lambda^{96}+\lambda^{4}+32.4 \lambda^{3}+530.9 \lambda^{2}+444.29 \lambda-4541$. The approximated solution of the characteristic equation is $\lambda_{390}=0.72$, whose argument is zero, which is the minimum argument, and hence the stability necessary condition becomes $\pi / 200-0>0$ which solves for $0.01571>0$. Hence, the FOSG system is stable and chaos exists in the incommensurate system.

5.3. Bicoherence of FOSG System. In Section 3.4, we have discussed the bicoherence of integer order smart grid. In this section we will investigate the bicoherence of FOSG 
system. The commensurate fractional order of the FOSG system is taken as 0.992 . As can be seen from Figures 10(a) and 10(b), compared to the integer order bicoherence contours (Figures 5 and 6), the bicoherence of FOSG systems shows multifrequency components contributing to the power spectrum. The multifrequency components can be seen denser in FOSG bicoherence confirming the existence of higher dimension spectrums. Also the same can be observed when investigating the bicoherence contours of all states together as seen from Figure 10(c). When compared with the integer order all state bicoherence (Figure 7), we can see that the fractional order bicoherence shows more yellow shades confirming the cross-bicoherence is significantly nonzero, and nonconstant, indicating a nonlinear relationship between the states. Hence by the above investigations we conclude that the nonlinearities occurring in the fractional order model of the smart grid system are more of the multifrequency nature and thus contributing to greater power spectrums compared to the integer order bicoherence.

\section{Adaptive Sliding Mode Control of Nonlinearity in FOSG}

The control goal of this paper is to design a suitable adaptive sliding mode controller for suppression of chaotic oscillations in the fractional order smart grid (FOSG) system (9). For deriving the robust ASMC controller for system (9), let us redefine the fractional order system with an adaptive sliding mode controller $u_{i}(t), i=1,2,3,4$,

$$
\begin{aligned}
& D^{q_{1}} x_{1}=x_{2}+u_{1} \text {, } \\
& D^{q_{2}} x_{2}=0.573-0.167 x_{2} \\
& +20 x_{4} \cos \left(x_{1}-x_{3}+1.483\right) \\
& +11.667 x_{4} \cos \left(x_{1}+1.483\right)+u_{2} \text {, } \\
& D^{q_{3}} x_{3}=69-93.33 x_{4}-179.05 x_{4}^{2}-50 \widehat{i_{0}} x_{4} \sin x_{3} \\
& -300 x_{4} \sin x_{3} \cos \left(x_{1}-1.483\right)+u_{3} \text {, } \\
& D^{q_{4}} x_{4}=25.322 x_{4}^{2}+13.054 x_{4} \\
& +3.529 x_{4} \cos \left(x_{1}-1.483\right) \\
& -3.529 x_{4} \cos x_{3} \cos \left(x_{3}-1.483\right) \\
& +42.353 x_{4} \sin x_{3} \cos \left(x_{1}-1.483\right) \\
& +7.059 \hat{i}_{0} x_{4} \sin x_{3} \\
& -42.353 x_{4} \sin x_{3} \cos \left(x_{3}-1.483\right) \\
& -35.294 x_{4} \sin \left(x_{3}+1.483\right) \\
& +0.588 \widehat{i}_{0} x_{4} \cos x_{3}
\end{aligned}
$$

$$
\begin{aligned}
& +2.941 x_{4} \cos \left(x_{3}-1.483\right)+1.31778 \\
& +u_{4} .
\end{aligned}
$$

The initial conditions are the same as in Section 2 and the parameter value $i_{0}$ is assumed unknown and is estimated with a parameter estimate $\widehat{i_{0}}$.

\subsection{Controller Design}

6.1.1. Problem Statement. In this section we investigate the adaptive fractional order sliding mode control (FOASMC) of a fractional order system. Let us define a generalized fractional order system with FOASMC as

$$
D^{q} x=f(x)+F(x) a+u(t),
$$

where $q$ is the fractional order of the system and where $u(t)$ is the adaptive controller for controlling the chaotic oscillations in the slave system.

Let us define the control error as

$$
e=0-x .
$$

The sliding surface for the fractional order integral sliding mode control $[40,41]$ is defined as

$$
s(e)=e+k \int e(\tau) d \tau .
$$

The fractional first derivative of the sliding surface is derived as

$$
D^{q} s=D^{q} e+k e .
$$

The fractional order error dynamics is defined as

$$
D^{q} e=-D^{q} x .
$$

Using (11) and (12) in (15)

$$
D^{q} e=-f(x)-F(x) a+u(t) .
$$

Let us define the adaptive controller as

$$
u(t)=f(x)+F(x) \widehat{a}-k e-\eta \operatorname{sgn}(s)-\rho s,
$$

where $k, \eta, \rho$ are positive gain values, $\widehat{a}$ is parameter estimate fractional order system, and $s$ is the sliding surface.

Using (17) in (16), the error dynamics simplifies to

$$
D^{q} e=-F(x)[a-\widehat{a}]-\eta \operatorname{sgn}(s)-\rho s .
$$

The Lyapunov candidate function to analyze the stability of the controller is defined as

$$
V=\frac{1}{2} s^{2}+\frac{1}{2}(a-\widehat{a})^{2} .
$$

The Lyapunov first derivate is derived as

$$
\dot{V}=s \cdot \dot{s}+(a-\widehat{a})(-\dot{\widehat{a}}) .
$$



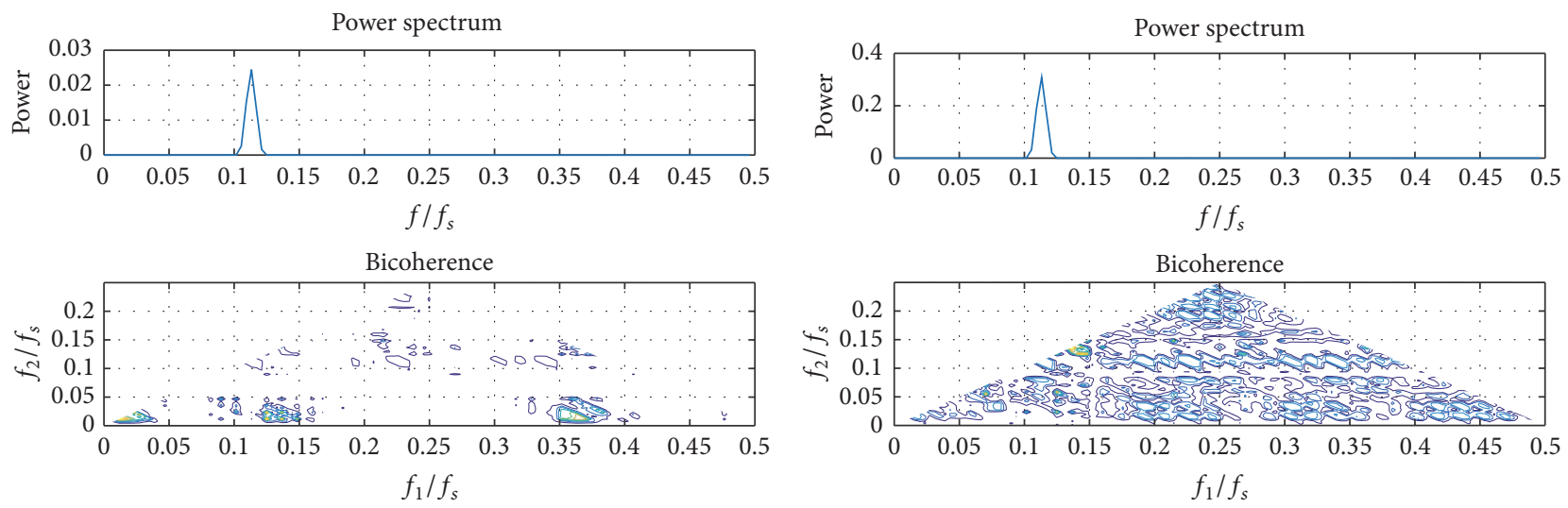

(a) Contour of bicoherence of state $x$ for the FOSG system

(b) Contour of bicoherence of state $y$ for the FOSG system
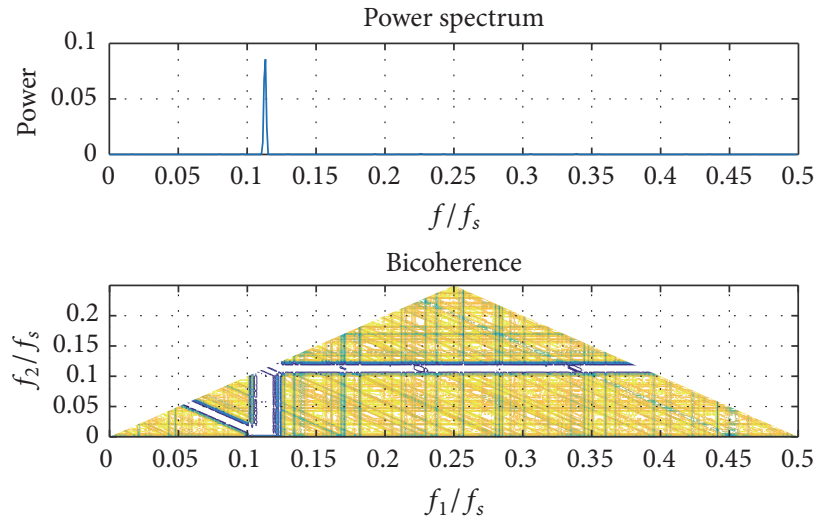

(c) Contour of bicoherence of all the states of the FOSG system

FIgURE 10: Bicoherence of FOSG system.

By definition of fractional calculus [42-44],

$$
\dot{x}(t)=D_{t}^{1-q} \cdot D_{t}^{q} x(t) .
$$

Using (21) in (20),

$$
\dot{V}=s \cdot D_{t}^{1-q} \cdot D_{t}^{q} s-(a-\widehat{a})\left(D_{t}^{1-q} \cdot D_{t}^{q} \widehat{a}\right) .
$$

Finding the sign of the Lyapunov first derivative using (22) seems difficult and hence we use the modified fractional order Lyapunov method defined by Rajagopal et al. [19] as

$$
\frac{1}{2} D_{t}^{q} x^{2}(t) \leq x(t) \frac{1}{2} D_{t}^{q} x(t), \quad q \in(0,1) .
$$

Using (23), (18), and (17) in (20),

$$
\begin{aligned}
\dot{V} \leq & k s[-F(x)(a-\widehat{a})-\eta \operatorname{sgn}(s)-\rho s] \\
- & (a-\widehat{a})\left(D^{q} \widehat{a}\right) .
\end{aligned}
$$

Let us define the parameter estimate laws as

$$
D^{q} \widehat{a}=k s \cdot F(x) .
$$

Using (25) in (24)

$$
\dot{V}=-\eta\left|s_{w}\right|-\rho s^{2}
$$

as $\eta$ and $\rho$ are all positive and $\dot{V}$ is negative definite. Using Barbalat's lemma [45], we conclude that $e(t) \rightarrow 0$ as $t \rightarrow \infty$.
6.1.2. Adaptive Sliding Mode Control of FOSG System. The main control objective of this paper is to design adaptive sliding mode controllers $\left(u_{1}, u_{2}, u_{3}, u_{4}\right)$ such that the chaotic oscillations in the FOSG system are controlled. Sliding mode control methodology is a simple approach to robust control and good at dealing with dynamic uncertainty [40]. The control design procedure consists of two steps: first constructing a sliding surface which presents the desired dynamics $[41,46]$ and second selecting a switching control law so as to verify sliding condition.

Let us define the integral sliding surface as

$$
s_{i}=x_{i}+k_{i} \int_{0}^{t} x_{i}(\tau) d \tau, \quad i=1,2,3,4 .
$$

The fractional derivate of the sliding surface is given by

$$
D^{q_{i}} s_{i}=D^{q_{i}} x_{i}+k_{i} x_{i}, \quad i=1,2,3,4 .
$$

For any initial conditions, the problem control of chaotic oscillations in FOSG system is equivalent to that of the states of the FOSG system remaining on the surface $s_{i}$ for all $t>0$. When the system operates in the sliding surface, it satisfies $s=0$ and $D^{q_{i}} s=0$ for $i=1,2,3,4$.

The parameter estimation errors are given by

$$
e_{i_{0}}=\hat{i}_{0}-i_{0}
$$


The fractional derivative of the parameter estimation errors is

$$
D^{q} e_{i_{0}}=D^{q} \widehat{i_{0}}-i_{0} .
$$

Let us the define the adaptive sliding mode controllers as

$$
\begin{aligned}
u_{1}= & -x_{2}-k_{1} x_{1}-\eta_{1} \operatorname{sgn}\left(s_{1}\right)-\rho_{1} s_{1}, \\
u_{2}= & -0.573+0.167 x_{2}-20 x_{4} \cos \left(x_{1}-x_{3}+1.483\right) \\
& -11.667 x_{4} \cos \left(x_{1}+1.483\right)-k_{2} x_{2} \\
& -\eta_{2} \operatorname{sgn}\left(s_{2}\right)-\rho_{2} s_{2}, \\
u_{3}= & -69+93.33 x_{4}+179.05 x_{4}^{2}+50 \widehat{\hat{i}_{0}} x_{4} \sin x_{3} \\
& +300 x_{4} \sin x_{3} \cos \left(x_{1}-1.483\right)-k_{3} x_{3} \\
& -\eta_{3} \operatorname{sgn}\left(s_{3}\right)-\rho_{3} s_{3}, \\
u_{4}= & -25.322 x_{4}^{2}-13.054 x_{4} \\
& -3.529 x_{4} \cos \left(x_{1}-1.483\right) \\
& +3.529 x_{4} \cos x_{3} \cos \left(x_{3}-1.483\right) \\
& -42.353 x_{4} \sin x_{3} \cos \left(x_{1}-1.483\right) \\
& -7.059 \widehat{i_{0}} x_{4} \sin x_{3} \\
& +42.353 x_{4} \sin x_{3} \cos \left(x_{3}-1.483\right) \\
& +35.294 x_{4} \sin \left(x_{3}+1.483\right) \\
& -0.588 \hat{i_{0}} x_{4} \cos x_{3} \\
& -2.941 x_{4} \cos \left(x_{3}-1.483\right)-1.31778-k_{4} x_{4} \\
& -\eta_{4} \operatorname{sgn}\left(s_{4}\right)-\rho_{4} s_{4},
\end{aligned}
$$

where $k_{i}, \rho_{i}, \eta_{i}$ for $i=1,2,3,4$ are positive constants and the parameter estimate of $i_{0}$ is

$$
\begin{aligned}
D^{q} \widehat{i_{0}}= & -50 s_{3} x_{4} \sin x_{3}+7.059 s_{4} x_{4} \sin x_{3} \\
& +0.588 s_{4} x_{4} \cos x_{3}-k e_{i_{0}},
\end{aligned}
$$

where $k$ is the estimation gain and is always positive.

\subsection{Stability Analysis of the Controller}

Theorem 1. Considering that adaptive sliding mode control input law in (15) is used to control the FOSG system in (10) with parameter estimate in (32), then the controller (31) is globally asymptotically stable.

Proof. To check the stability of the controlled system, let us consider the following Lyapunov candidate function:

$$
V=\frac{1}{2}\left[s_{1}^{2}+s_{2}^{2}+s_{3}^{2}+s_{4}^{2}+e_{i_{0}}^{2}\right] .
$$

The first derivative of the Lyapunov candidate function is

$$
\dot{V}=s_{1} \dot{s}_{1}+s_{2} \dot{s}_{2}+s_{3} \dot{s}_{3}+s_{4} \dot{s}_{4}+e_{i_{0}} \dot{e}_{i_{0}} .
$$

By definition of fractional calculus [42-44], we obtain

$$
\dot{x}(t)=D_{t}^{1-q} \cdot D_{t}^{q} x(t) .
$$

Applying (35) in (34)

$$
\begin{aligned}
\dot{V}= & s_{1} D_{t}^{1-q_{1}} \cdot D_{t}^{q_{1}} s_{1}+s_{2} D_{t}^{1-q_{2}} \cdot D_{t}^{q_{2}} s_{2}+s_{3} D_{t}^{1-q_{3}} \\
& \cdot D_{t}^{q_{3}} s_{3}+s_{4} D_{t}^{1-q_{4}} \cdot D_{t}^{q_{4}} s_{4}+e_{i_{0}} D_{t}^{1-q} \cdot D_{t}^{q} e_{i_{0}} .
\end{aligned}
$$

Thus, it is clear that stability calculations with (36) are very difficult. So, we use modified Lyapunov stability theory as given in [19].

Let $x(t)$ be a time continuous and derivable function. As proved in [19], for any time instant $t \geq t_{0}$, we have

$$
\frac{1}{2} D_{t}^{q} x^{2}(t) \leq x(t) \cdot D_{t}^{q} x(t) \quad \forall q \in(0,1) .
$$

Using (37) in (34)

$$
\begin{aligned}
\dot{V} \leq & s_{1}\left[D^{q_{1}} x_{1}+k_{1} x_{1}\right]+s_{2}\left[D^{q_{2}} x_{2}+k_{2} x_{2}\right] \\
& +s_{3}\left[D^{q_{3}} x_{3}+k_{3} x_{3}\right]+s_{4}\left[D^{q_{4}} x_{4}+k_{4} x_{4}\right] \\
& +e_{i_{0}} D^{q_{i_{0}}} .
\end{aligned}
$$

Using the ASM controllers (31) and parameter estimate (32) in (38),

$$
\begin{aligned}
\dot{V} \leq & -\eta_{1}\left|s_{1}\right|-\eta_{2}\left|s_{2}\right|-\eta_{3}\left|s_{3}\right|-\eta_{4}\left|s_{4}\right|-\rho_{1} s_{1}^{2}-\rho_{2} s_{2}^{2} \\
& -\rho_{3} s_{3}^{2}-\rho_{4} s_{4}^{2} .
\end{aligned}
$$

As $\rho_{i}$ and $\eta_{i}$ are positive for $i=1,2,3,4$, using Barbalat's lemma [45], the Lyapunov first derivative (39) is a negative definite function which infers that the controller is stable and is valid for any bounded initial conditions.

\section{Numerical Simulations}

The FOSG system (10) with the robust adaptive sliding mode controller (31) and parameter estimate (32) with initial conditions as in Section 2 and initial condition for the parameter estimate $i_{0}(0)=0.1$ are numerically analyzed using Matlab. The fractional orders of the FOSG system are chosen as commensurate order $q=0.992$. The controller gains are taken as $k_{i}=10$ and sliding surface gains are taken as $\rho_{i}=25$ and $\eta_{i}=0.1$ with $i=1,2,3,4$.

The state trajectories of the controlled fractional order induction chaotic system (10) are shown in Figure 11(a). Figure 11(b) shows the parameter estimate with controller in action. It can be clearly observed that the state trajectories converge to zero as soon as the controller is introduced which clearly shows that the fractional order system (9) is wellcontrolled by the adaptive controller with the uncertainty in the inverter current $i_{0}$. 


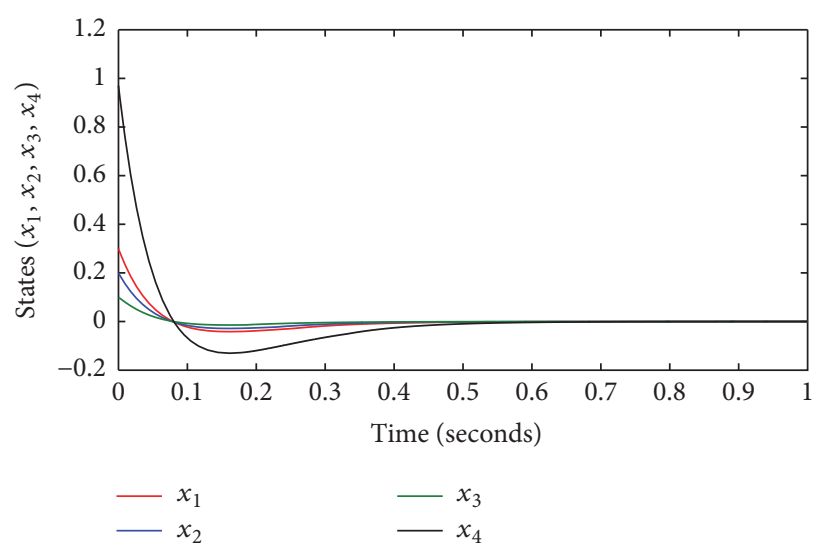

(a) Time history of the controlled states of FOSG system

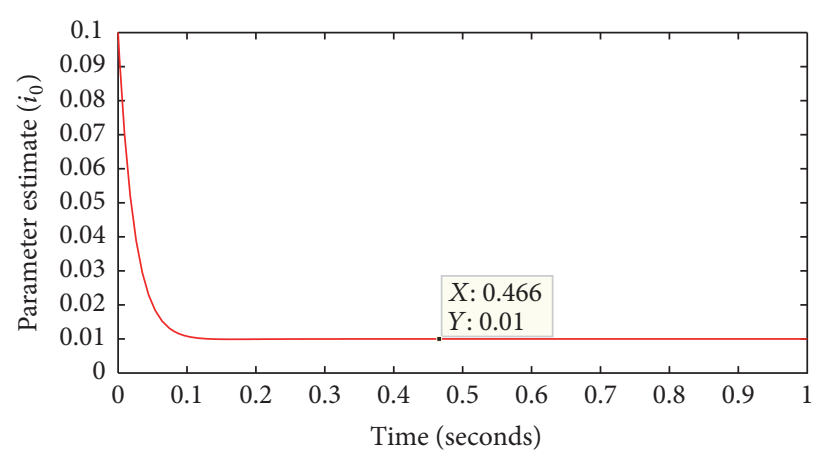

(b) Time history of parameter estimate $\hat{i_{0}}$

Figure 11

\section{Chaos Control with Genetically Optimized Fractional Order PID Controllers (GAFOPID)}

Fractional order PID (FOPID) controllers described by using fractional calculus are the most common and useful algorithm in control systems engineering. In most cases, feedback loops are controlled using PID algorithms; they are designed to correct error(s) between instant value(s) in a system and chosen set point values. Design of FOPID controller using genetic algorithms (GA) which is a stochastic global search method uses the process of natural evolution. It is one of the methods used for optimization and successfully applied in $[47,48]$. GA have to be initialized before the algorithm can proceed. The initialization of the population size, variable bounds, and the evaluation objective functions are required, to evaluate the best gain values of FOPID controller for the system. An objective function could be created to find a FOPID controller that gives a minimal error. The error functions such as sum absolute error (SAE) are used as objective functions in this work.

Let us define the fractional order PID controllers as

$$
u_{i}=K_{\mathrm{P}} e_{i}+K_{\mathrm{I}} \int_{0}^{t} e_{i} d \tau^{\beta}+K_{\mathrm{D}} \frac{d^{\delta} e_{i}}{d t^{\delta}}, \quad i=1,2,3,4,
$$

where $u_{i}$ is the fractional order PID action control, $\delta, \beta$ are the fractional order differential and integral operators [42-44], $e_{i}$ is the error signal given by $e_{i}=0-x_{i}$, and $K_{\mathrm{P}}, K_{\mathrm{I}}, K_{\mathrm{D}}$ are the proportional, integral, and the derivative gains to suppress the nonlinearity in the FOSG system.

Matlab optimization tool box is used for numerical simulation with the following options:

Variable bounds matrix of the proportional, integral, and the derivative gains $=[-0.001,0.001]$, but for the states $x_{3}, x_{4}$ the values are multiplied to hundred and ten for the difference of variation range.

Population size $=100$, GA. Generally the bigger the population size, the better the final approximation.

Number of generations $=100$.
TABLE 1: FOPID controller gain values optimized with GA.

\begin{tabular}{lccc}
\hline FOPID controller & $K_{\mathrm{P}}$ & $K_{\mathrm{I}}$ & $K_{\mathrm{D}}$ \\
\hline$u_{1}$ & 0.0601 & 0.0975 & 0.2522 \\
$u_{2}$ & 0.0830 & 0.1151 & 0.1719 \\
$u_{3}$ & 0.0081 & 0.3316 & 0.0417 \\
$u_{4}$ & -0.0365 & 0.2448 & 0.2107 \\
\hline
\end{tabular}

Selection function $=$ stochastic uniform.

Crossover fraction $=0.8$.

Mutation function $=$ Gaussian .

Stopping criteria $=$ error performance criterion.

Length of the chromosome $=12$, decimal coding.

The objective function is written based on error performance criterion sum absolute error as

$$
\text { fitness }=\frac{1}{\sum\left(e_{i}\right)}, \quad i=1,2,3,4 .
$$

Table 1 shows the FOPID gain values after running the GA solver from the optimization tool with the options cited above, we get the best solutions tracked over generations for the complete chaos suppression of the FOSG system via fractional order PID controllers using gains values genetically optimized; Figure 12 shows the time history of the FOSG states $\left(x_{1}, x_{2}, x_{3}, x_{4}\right)$ and Figure 13 shows the time history of parameter estimate.

As seen from Figures 10 and 12, in the fractional order adaptive sliding mode controller (FOASMC) method of control the states converge at $t=0.462 \mathrm{~s}$ whereas in the GAFOPID based control, the states converge at $t=0.22 \mathrm{~s}$. The states control in Figures 10 and 12 and the parameter estimation plots shown in Figures 11 and 13 clearly indicate that GAFOPID controllers are efficient compared to the FOASMC controllers. As can be seen from Figures 11 and 13, FOASMC based parameter estimation $(t=0.2521 \mathrm{~s})$ is slower than the GAFOPID controllers $(t=0.1746 \mathrm{~s})$. This proves that the optimized controllers are better in performance 


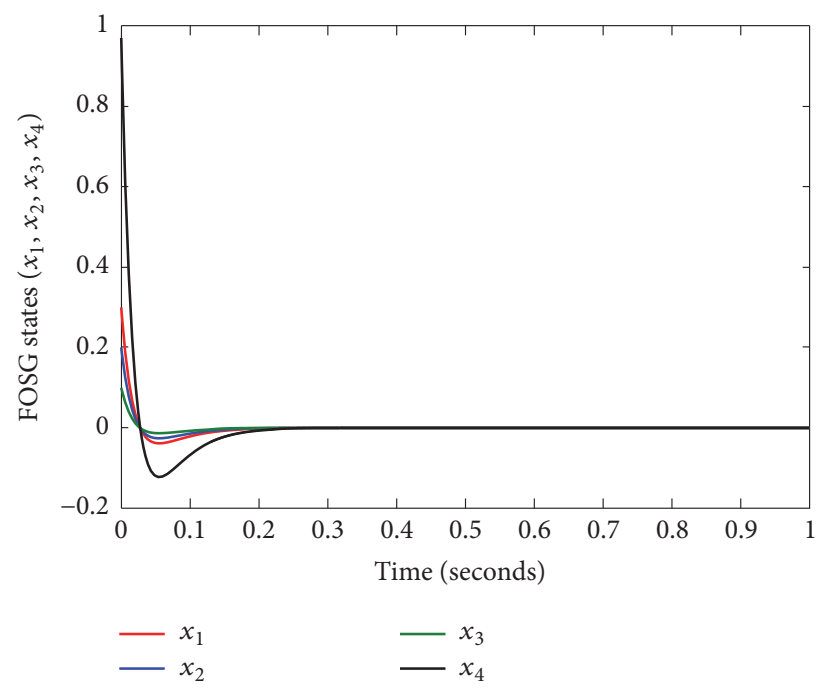

FIGURE 12: Time history of GAFOPID controlled FOSG system states.

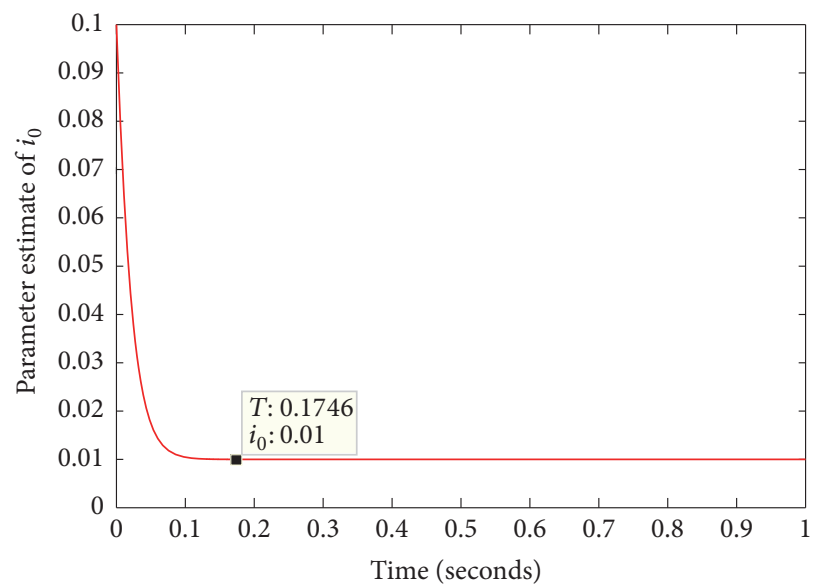

FIGURE 13: Time history of GAFOPID controlled parameter estimates.

than sliding mode controllers in the case of nonlinearity suppression in fractional order systems.

\section{FPGA Implementation of the FOSG System and GAFOPID Controllers}

9.1. FPGA Implementation of the FOSG System. In this section we discuss the implementation of the proposed fractional order smart grid system in FPGA [49-54] using the Xilinx (Vivado) System Generator toolbox in Simulink. Firstly we configure the available built-in blocks of the System Generator toolbox. The Add/Sub blocks are configured with zero latency and 32/16-bit fixed point settings. The output of the block is configured to rounded quantization in order to reduce the bit latency. For the multiplier block a latency of 3 is configured and the other settings are the same as in Add/Sub block. Next we will have to design the fractional order integrator which is not a readily available block in the System Generator [49]. Hence we implement the integrators using the mathematical relation discussed in [50] and the value of $h$ is taken as 0.001 and the initial conditions are fed into the forward register with fractional order taken as $q=0.992$ for FOSG system. The sinusoidal functions are implemented using the CORDIC 6.0 blocks and Figures 14, 15, 16(a), and 16(b) show the Xilinx RTL schematics of the FOSG system implemented in Kintex 7 (device = $7 \mathrm{k} 160 \mathrm{t}$; package $=\mathrm{fbg} 484 \mathrm{~S}$ ) and $3 \mathrm{D}$ phase portraits of the FPGA implemented FOSG system, power utilized by the system and power utilized for various fractional orders, respectively. Table 2 shows the resources utilized by the FOSG system including the clock frequency. Increasing the sampling time period in some implementations may lead to a clock frequency mismatch and hence plays a critical role in implementation. Also negative time slack may also create problems while implementing the design and hence choosing constraints may also be critical in cases where the number of logical operations is more. Also avoiding DDR clocks helps in reducing the route delays. For analyzing the power consumed by the controllers, we use the approximation methodology discussed in $[53,54]$. It confirms that larger 


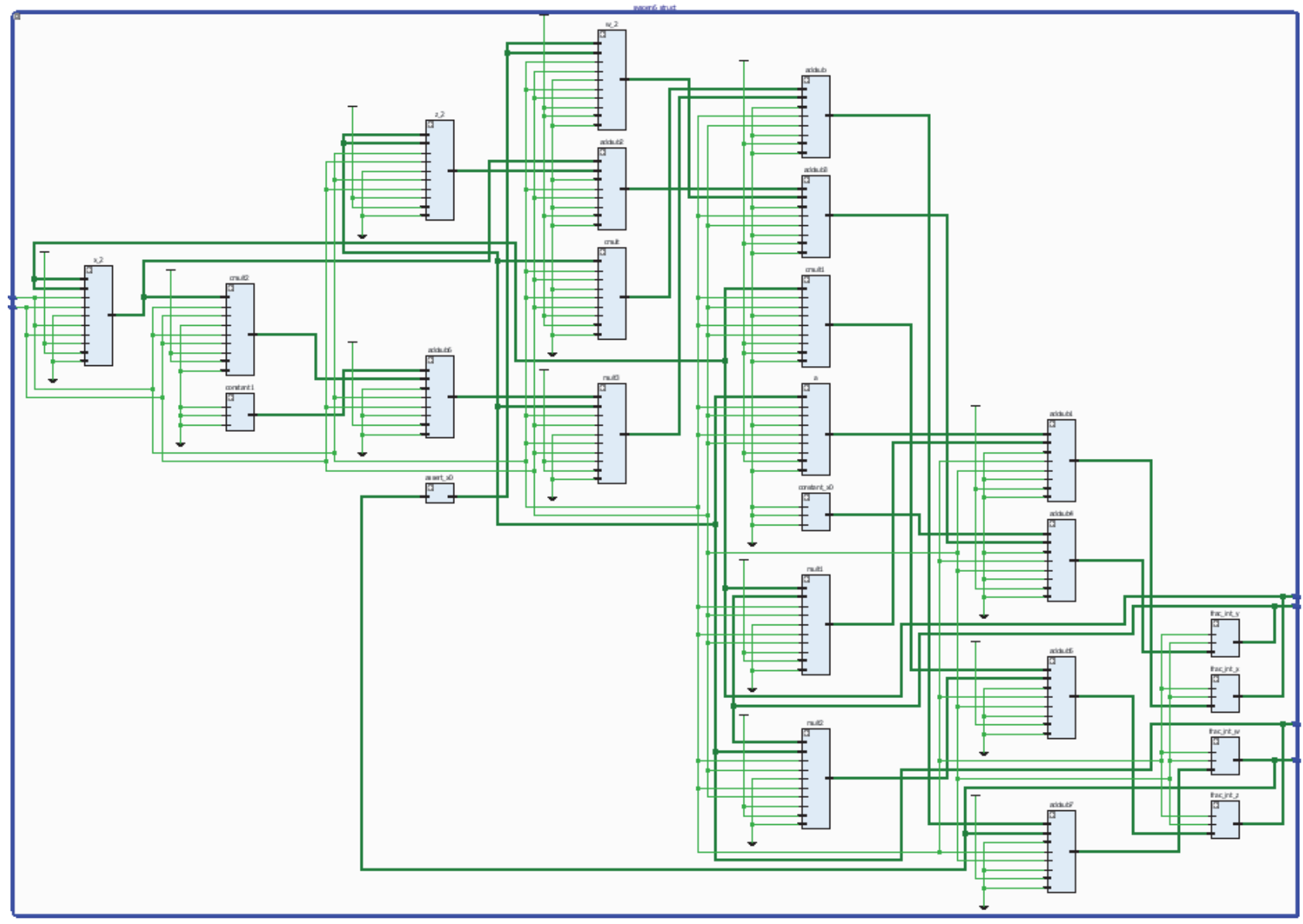

FIGURE 14: Xilinx schematics of the FOSG system implemented in Kintex 7 (device = 7k160t; package = fbg484 S).
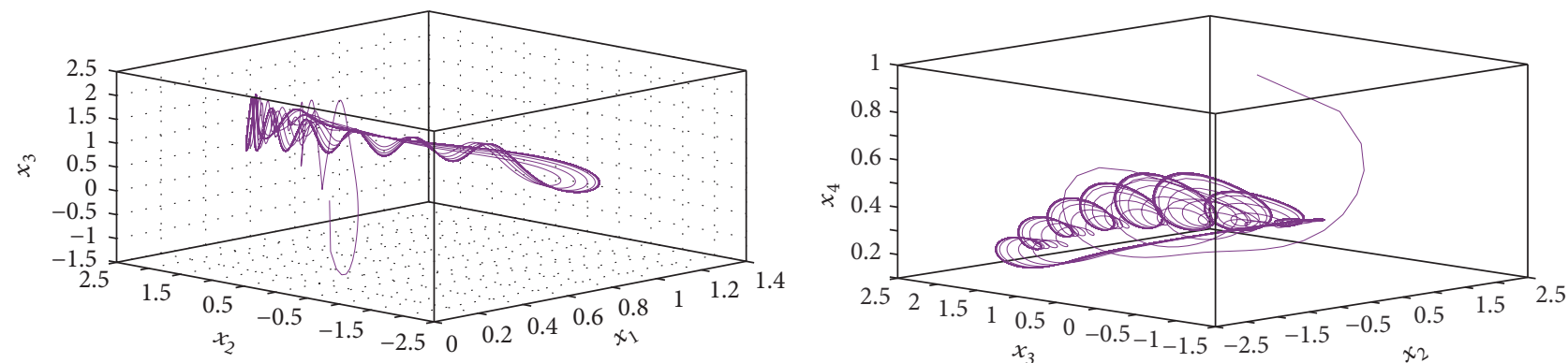

FIGURE 15: 3D phase portraits of the FPGA implemented FOSG system.

TABLE 2: Resources utilized by the FOSG system.

\begin{tabular}{|c|c|c|c|c|c|}
\hline \multirow{2}{*}{ Kintex 7 k160t } & \multirow{2}{*}{ Utilization } & \multirow{2}{*}{ Available } & \multirow{2}{*}{ Utilization\% } & \multicolumn{2}{|c|}{ Clock frequency } \\
\hline & & & & $f_{\max }$ & Used \\
\hline LUT & 1027 & 101400 & 1.01 & $500 \mathrm{Mhz}$ & $311 \mathrm{Mhz}$ \\
\hline FF & 954 & 202800 & 0.47 & $300 \mathrm{Mhz}$ & $160 \mathrm{Mhz}$ \\
\hline DSP & 51 & 600 & 8.5 & $500 \mathrm{Mhz}$ & $352 \mathrm{Mhz}$ \\
\hline $\mathrm{IO}$ & 132 & 285 & 46.3 & $250 \mathrm{Mhz}$ & $161 \mathrm{Mhz}$ \\
\hline BUFG & 3 & 32 & 9.37 & $300 \mathrm{Mhz}$ & $127 \mathrm{Mhz}$ \\
\hline
\end{tabular}




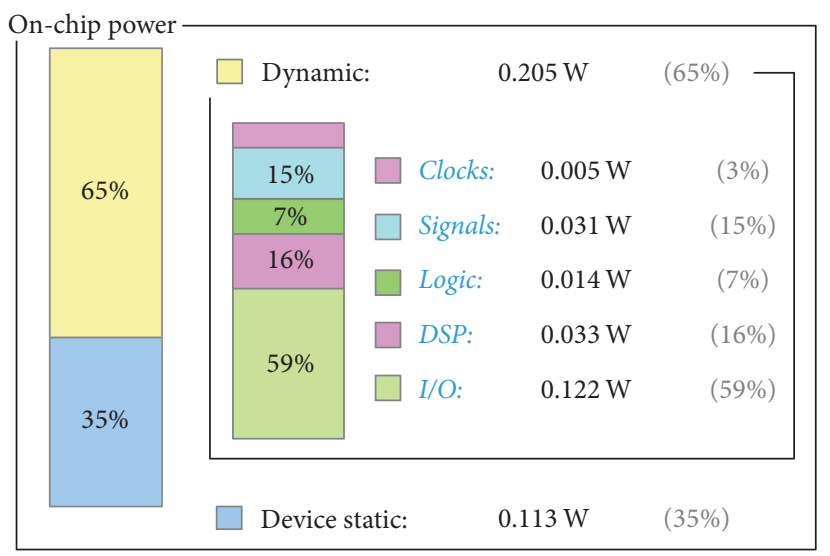

(a)

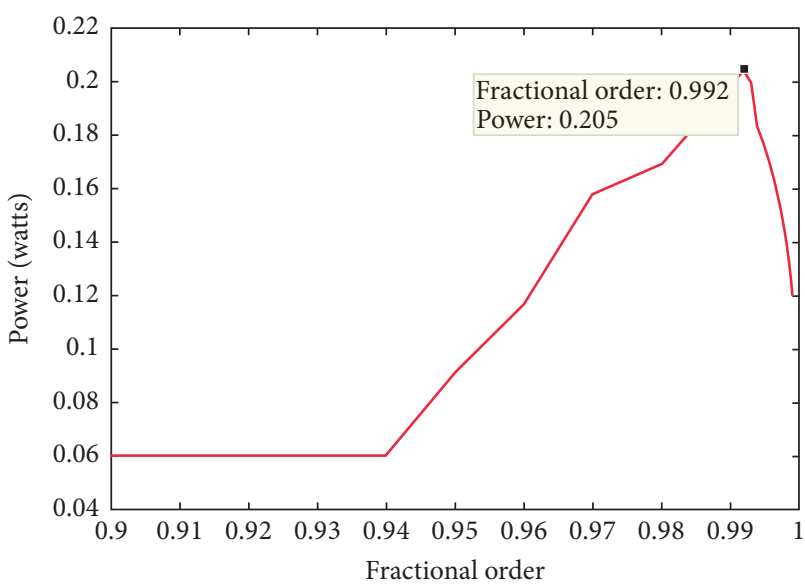

(b)

Figure 16: (a) Power utilized by FOSG system. (b) Power utilized for various fractional orders.

TABLE 3: Resource utilization of GAFOPID controllers.

\begin{tabular}{|c|c|c|c|c|c|}
\hline \multirow{2}{*}{ Kintex 7 k160t } & \multirow{2}{*}{ Utilization } & \multirow{2}{*}{ Available } & \multirow{2}{*}{ Utilization\% } & \multicolumn{2}{|c|}{ Clock frequency } \\
\hline & & & & $f_{\max }$ & Used \\
\hline LUT & 1124 & 101400 & 1.11 & $500 \mathrm{Mhz}$ & $339 \mathrm{Mhz}$ \\
\hline $\mathrm{FF}$ & 850 & 202800 & 0.42 & $300 \mathrm{Mhz}$ & $140 \mathrm{Mhz}$ \\
\hline DSP & 36 & 600 & 6.00 & $500 \mathrm{Mhz}$ & $344 \mathrm{Mhz}$ \\
\hline $\mathrm{IO}$ & 129 & 285 & 45.26 & $250 \mathrm{Mhz}$ & $129 \mathrm{Mhz}$ \\
\hline BUFG & 1 & 32 & 3.13 & $300 \mathrm{Mhz}$ & $112 \mathrm{Mhz}$ \\
\hline
\end{tabular}

power will be consumed by the system when the FOSG system shows largest Lyapunov exponents $(q=0.992)$. To utilize the power of FPGA, the computation needs to be divided into several independent blocks of threads that can be executed simultaneously [51]. The performance on FPGA is directly related to the number of threads and its performances decrease when number of branching instructions increases. Hence we designed the system and the GAFOPID controllers as four parallel threads each as shown in Figures 18 and 19. The fractional order operators are implemented as building blocks and the so-called frame delay is not noticeable in the FPGA hardware implementation due to its parallel data structure, unlike a microprocessor-based implementation. While FPGA implementations have a reputation for being difficult to design, with the help of systematic methodology, a system can be put together with less work than is required for more traditional software-based realizations [52].

9.2. FPGA Implementation of GAFOPID Controllers. In this section we implement the proposed fractional order genetically optimized PID controllers (GAFOPID) derived in (15) along with the fractional parameter update law (16). For implementation of the entire control scheme we use Kintex 7 (device $=7 \mathrm{k} 160 \mathrm{t}$; package $=\mathrm{fbg} 484 \mathrm{~S})$. The fractional order of the FOSG system and GAFOPID controllers are kept as $q=0.992$. For analyzing the power consumed by the controllers, we use the approximation methodology discussed in [54]. It confirms that larger power will be consumed by the controller when the FOSG system shows the largest Lyapunov exponent. Figure 17 shows the RTL schematics of the GAFOPID controllers implemented in Kintex 7 (device $=7 \mathrm{k} 160 \mathrm{t}$; package $=\mathrm{fbg} 484 \mathrm{~S}$ ). Figures 18(a) and 18(b) show the power utilization of the controller and power utilization with change in fractional orders, respectively. Table 3 shows the resources utilized by the GAFOPID controllers. Figure 19 shows the time history of the FOSG states controlled with FPGA implemented GAFOPID controllers. Figure 20 shows the time history of parameter estimates. The initial conditions for the numerical analysis are taken as in Section 7.

\section{Conclusion}

In this work we investigated the nonlinearity in a specific smart grid system. We showed the existence of chaotic oscillations for specific initial conditions and inverter current value. Fractional order smart grid dimensionless model of the smart grid is derived and investigated. Bifurcation of the system with fractional orders is investigated and it is shown that the largest Lyapunov exponent of the system exists in the fractional order. Hence fractional order control schemes for suppression of chaotic oscillations with fractional order adaptive sliding mode control (FOASMC) and genetically optimized fractional order PID control (GAFOPID) are derived. Numerical simulations prove that GAFOPID controllers are effective compared to FOASMC for chaos control 


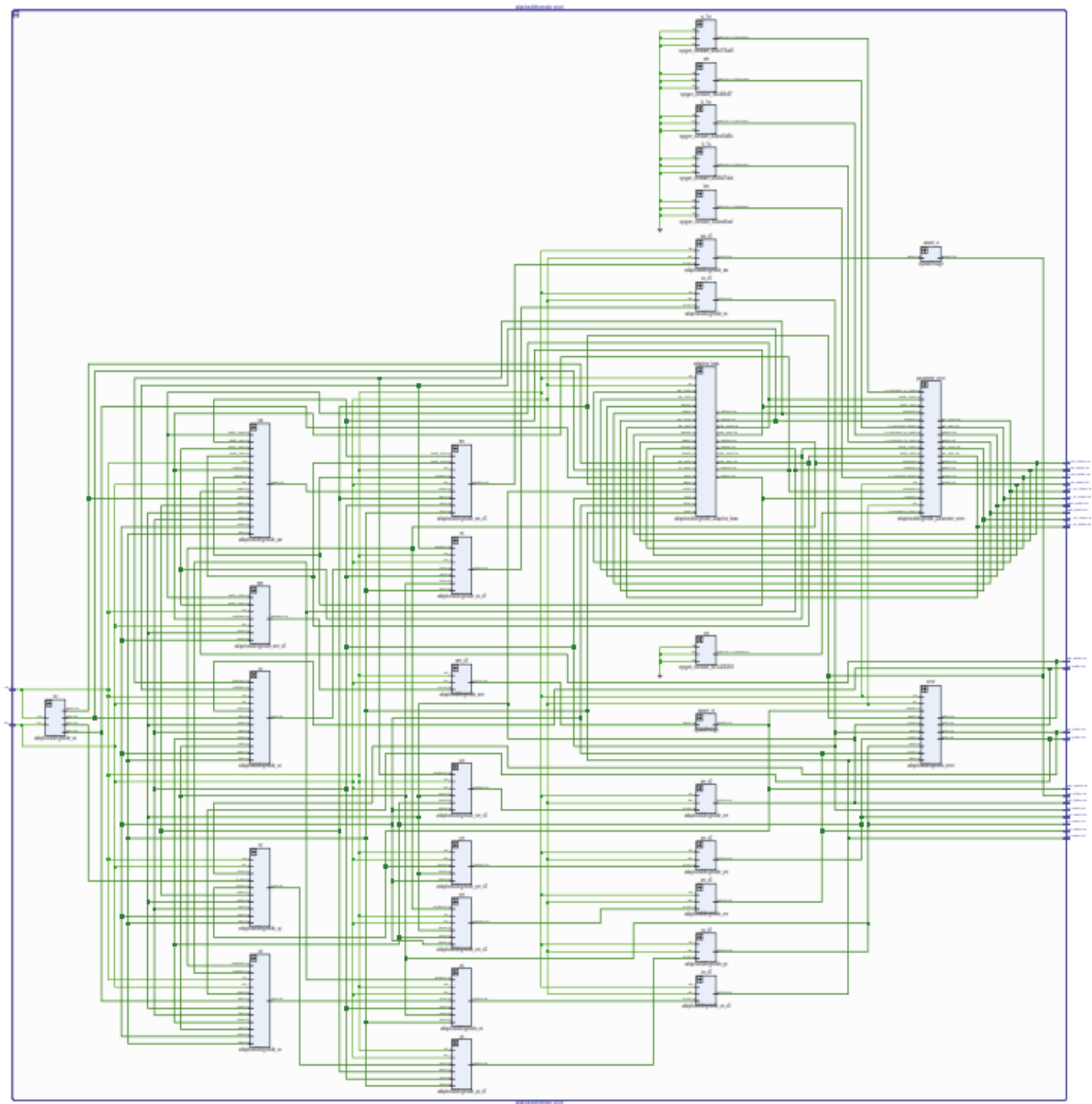

FIGURE 17: Xilinx schematics of the GAFOPID controllers implemented in Kintex 7 (device = 7k160t; package = fbg484 S).

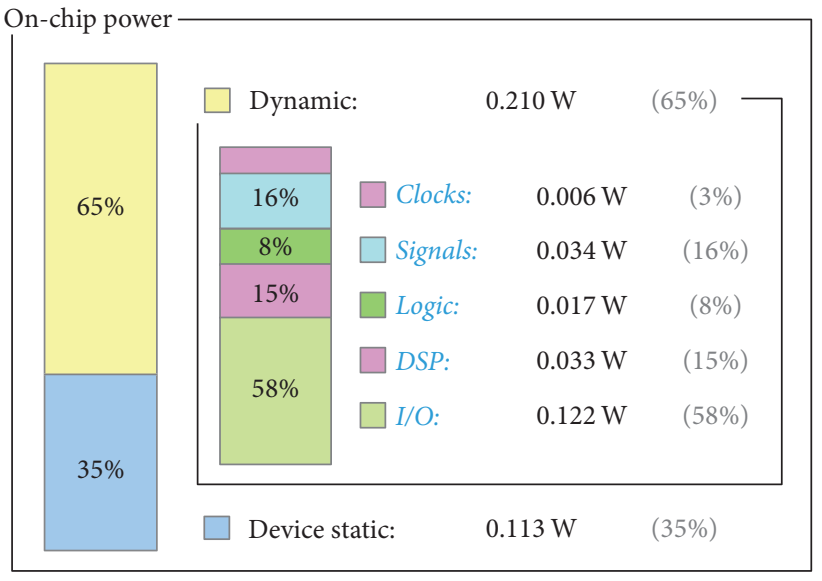

(a)

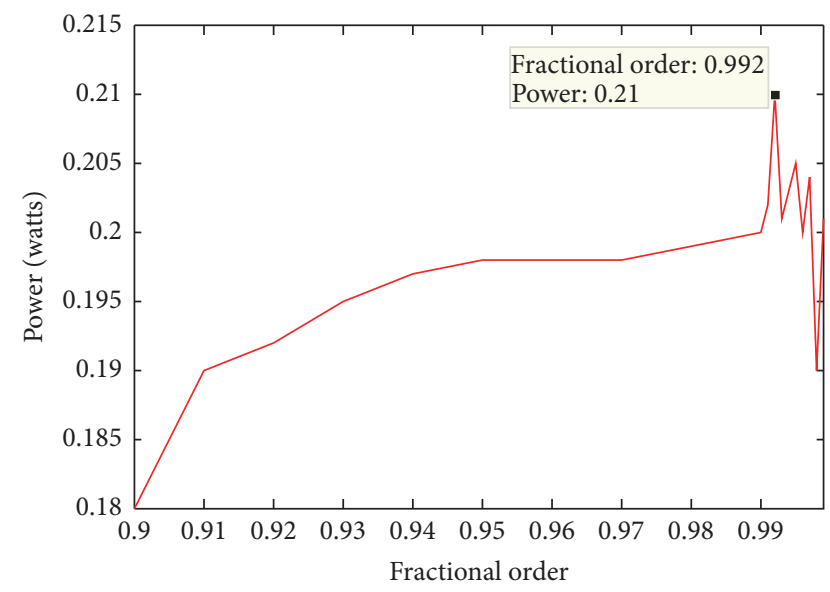

(b)

FIGURE 18: (a) Power utilized and (b) power utilization versus fractional order of GAFOPID controllers. 


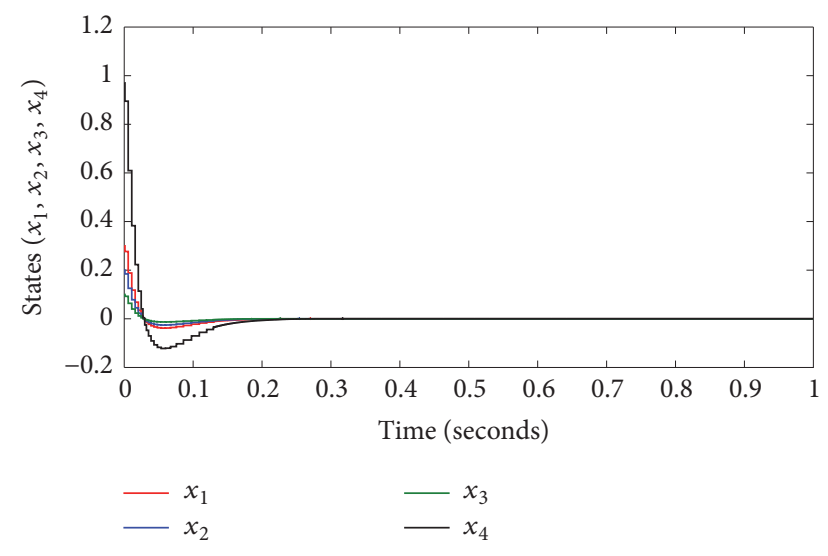

FIGURE 19: Time history of FOSG states controlled with FPGA implemented GAFOPID controllers.

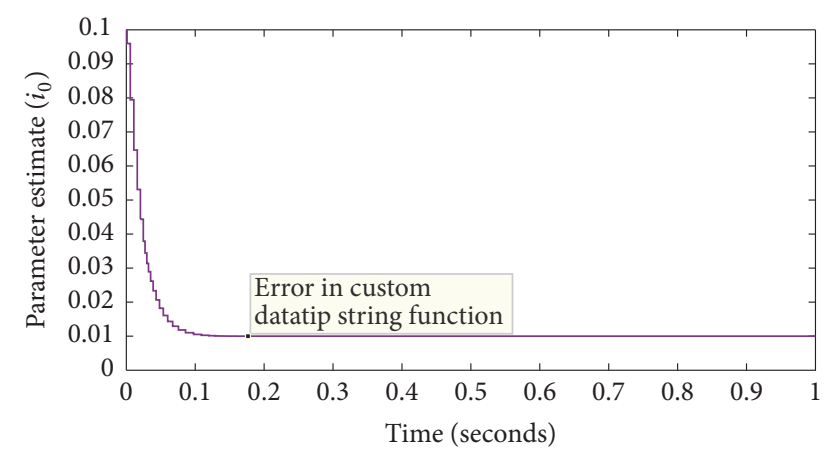

FIGURE 20: Time history of parameter estimates.

in fractional order systems. The proposed GAFOPID controllers are implemented in FPGA to show that the control scheme is hardware realizable. Power utilization for various fractional orders is presented and it is shown that the system uses maximum power when the FOSG system exhibits its largest Lyapunov exponent.

\section{Conflicts of Interest}

The authors declare that there are no conflicts of interest regarding the publication of this paper.

\section{References}

[1] R. Tongia, "Ph.D. Smart Grids White Paper: WH-1:14.8," https:// cstep.in/node/47.

[2] K. Moslehi and R. Kumar, "A reliability perspective of the smart grid," IEEE Transactions on Smart Grid, vol. 1, no. 1, pp. 57-64, 2010.

[3] US Department of Energy, How the Smart Grid Promotes a Greener Future, 2010, http://energy.gov/oe/downloads/howsmart-grid-promotes-greener-future.

[4] J. Xia and Y. Wang, "Secure key distribution for the smart grid," IEEE Transactions on Smart Grid, vol. 3, no. 3, pp. 1437-1443, 2012.
[5] C. Canizares, "Voltage stability assessment, procedures and guides," IEEE/PES Power System Stability Subcommittee Special Publication, 2001.

[6] W. Qiao, G. K. Venayagamoorthy, and R. G. Harley, "Missingsensor-fault-tolerantcControl for SSSC fACTS device with realtime implementation," IEEE Transactions on Power Delivery, vol. 24, no. 2, pp. 740-750, 2009.

[7] S. Vaidyanathan and C. Volos, Advances and Applications of Chaotic Systems, Springer, Berlin, Germany, 2016.

[8] N. Hemati and M. C. Leu, "A complete model characterization of brushless DC motors," IEEE Transactions on Industry Applications, vol. 28, no. 1, pp. 172-180, 1992.

[9] Z. Li, J. B. Park, Y. H. Joo, B. Zhang, and G. Chen, "Bifurcations and chaos in a permanent-magnet synchronous motor," IEEE Transactions on Circuits and Systems I: Fundamental Theory and Applications, vol. 49, no. 3, pp. 383-387, 2002.

[10] Z. Jing, C. Yu, and G. Chen, "Complex dynamics in a permanent-magnet synchronous motor model," Chaos, Solitons \& Fractals, vol. 22, no. 4, pp. 831-848, 2004.

[11] N. Jabli, H. Khammari, M. F. Mimouni, and R. Dhifaoui, "Bifurcation and chaos phenomena appearing in induction motor under variation of PI controller parameters," WSEAS Transactions on Systems, vol. 9, no. 7, pp. 784-793, 2010.

[12] H. Gholizadeh, A. Hassannia, and A. Azarfar, "Chaos detection and control in a typical power system," Chinese Physics B, vol. 22, no. 1, Article ID 010503, 2013.

[13] H.-K. Chen, T.-N. Lin, and J.-H. Chen, "Dynamic analysis, controlling chaos and chaotification of a SMIB power system," Chaos, Solitons and Fractals, vol. 24, no. 5, pp. 1307-1315, 2005.

[14] M. P. Aghababa, "Robust finite-time stabilization of fractionalorder chaotic systems based on fractional lyapunov stability theory," Journal of Computational and Nonlinear Dynamics, vol. 7, no. 2, pp. 21-31, 2012.

[15] E. A. Boroujeni and H. R. Momeni, "Non-fragile nonlinear fractional order observer design for a class of nonlinear fractional order systems," Signal Processing, vol. 92, no. 10, pp. 2365-2370, 2012.

[16] R. Zhang and J. Gong, "Synchronization of the fractional-order chaotic system via adaptive observer," Systems Science \& Control Engineering, vol. 2, no. 1, pp. 751-754, 2014.

[17] I. Petras, "Methos for simulation of the fractional order chaotic systems," Acta Montanastica Slovaca, vol. 11, no. 4, pp. 273-277, 2006.

[18] Z. W. Trzaska, Matlab Solutions of Chaotic Fractional Order Circuits, InTech, Rijeka, Croatia, 2013, https://cdn.intechopen .com/pdfs-wm/21404.pdf.

[19] K. Rajagopal, S. Vaidyanathan, A. Karthikeyan, and P. Duraisamy, "Dynamic analysis and chaos suppression in a fractional order brushless DC motor," Electrical Engineering, 2016.

[20] K. Rajagopal, A. Karthikeyan, and P. Duraisamy, "Chaos suppression in fractional order permanent magnet synchronous motor and PI controlled induction motor by extended back stepping control," Nonlinear Engineering, vol. 5, no. 4, 2016.

[21] C.-L. Li, S.-M. Yu, and X.-S. Luo, "Fractional-order permanent magnet synchronous motor and its adaptive chaotic control," Chinese Physics B, vol. 21, no. 10, Article ID 100506, 2012.

[22] Y. Li, Y. Chen, and I. Podlubny, "Stability of fractional-order nonlinear dynamic systems: Lyapunov direct method and generalized Mittag-LEFfler stability," Computers \& Mathematics with Applications, vol. 59, no. 5, pp. 1810-1821, 2010. 
[23] J. A. Gallegos and M. A. Duarte-Mermoud, "On the Lyapunov theory for fractional order systems," Applied Mathematics and Computation, vol. 287-288, pp. 161-170, 2016.

[24] M. S. Tavazoei and M. Haeri, "Chaos control via a simple fractional-order controller," Physics Letters A, vol. 372, no. 6, pp. 798-807, 2008.

[25] K. Konishi, H. Kokame, and N. Hara, "Delayed feedback control based on the act-and-wait concept," Nonlinear Dynamics, vol. 63, no. 3, pp. 513-519, 2011.

[26] Y. Jin, Y.-Q. Chen, and D. Xue, “Time-constant robust analysis of a fractional order [proportional derivative] controller," IET Control Theory and Applications, vol. 5, no. 1, pp. 164-172, 2011.

[27] K. Rajagopal, L. Guessas, S. Vaidyanathan, A. Karthikeyan, and A. Srinivasan, "Dynamical analysis and FPGA implementation of a novel hyperchaotic system and its synchronization using adaptive sliding mode control and genetically optimized PID Control," Mathematical Problems in Engineering, vol. 2017, Article ID 7307452, 14 pages, 2017.

[28] K. Rajagopal, A. Karthikeyan, and A. K. Srinivasan, "FPGA implementation of novel fractional-order chaotic systems with two equilibriums and no equilibrium and its adaptive sliding mode synchronization," Nonlinear Dynamics, vol. 87, no. 4, pp. 2281-2304, 2017.

[29] E. Tlelo-Cuautle, A. D. Pano-Azucena, J. J. Rangel-Magdaleno, V. H. Carbajal-Gomez, and G. Rodriguez-Gomez, "Generating a 50 -scroll chaotic attractor at $66 \mathrm{MHz}$ by using FPGAs," Nonlinear Dynamics, vol. 85, no. 4, pp. 2143-2157, 2016.

[30] Q. Wang, S. Yu, C. Li et al., "Theoretical design and FPGA-based implementation of higher-dimensional digital chaotic systems," IEEE Transactions on Circuits and Systems. I. Regular Papers, vol. 63, no. 3, pp. 401-412, 2016.

[31] E. Dong, Z. Liang, S. Du, and Z. Chen, “Topological horseshoe analysis on a four-wing chaotic attractor and its FPGA implement," Nonlinear Dynamics, vol. 83, no. 1-2, pp. 623-630, 2016.

[32] E. Tlelo-Cuautle, V. H. Carbajal-Gomez, P. J. Obeso-Rodelo, J. J. Rangel-Magdaleno, and J. C. Nunez-Perez, "FPGA realization of a chaotic communication system applied to image processing," Nonlinear Dynamics, vol. 82, no. 4, pp. 1879-1892, 2015.

[33] V. Rashtchi and M. Nourazar, "FPGA implementation of a realtime weak signal detector using a duffing oscillator," Circuits, Systems, and Signal Processing, vol. 34, no. 10, pp. 3101-3119, 2015.

[34] E. Tlelo-Cuautle, J. J. Rangel-Magdaleno, A. D. Pano-Azucena, P. J. Obeso-Rodelo, and J. C. Nunez-Perez, "FPGA realization of multi-scroll chaotic oscillators," Communications in Nonlinear Science and Numerical Simulation, vol. 27, no. 1-3, pp. 66-80, 2015.

[35] Y.-M. Xu, L.-D. Wang, and S.-K. Duan, "A memristor-based chaotic system and its field programmable gate array implementation," Acta Physica Sinica, vol. 65, no. 12, Article ID 120503, 2016.

[36] Q. Sun, Y. Wang, J. Yang, Y. Qiu, and H. Zhang, "Chaotic dynamics in smart grid and suppression scheme via generalized fuzzy hyperbolic model," Mathematical Problems in Engineering, vol. 2014, Article ID 761271, 2014.

[37] J. M. Munoz-Pacheco, L. d. Gómez-Pavón, O. G. Félix-Beltrán, and A. Luis-Ramos, "Determining the Lyapunov spectrum of continuous-time $1 \mathrm{D}$ and 2D multiscroll chaotic oscillators via the solution of m-PWL variational equations," Abstract and Applied Analysis, vol. 2013, Article ID 851970, 11 pages, 2013.

[38] A. Wolf, J. B. Swift, H. L. Swinney, and J. A. Vastano, "Determining Lyapunov exponents from a time series," Physica D: Nonlinear Phenomena, vol. 16, no. 3, pp. 285-317, 1985.
[39] C. Pezeshki, "Bispectral analysis of [systems] possessing chaotic motion," Journal of Sound and Vibration, vol. 137, no. 3, pp. 357368, 1990.

[40] K. Rajagopal and A. Karthikeyan, "Chaos suppression of fractional order willamowski-rössler chemical system and its synchronization using sliding mode control," Nonlinear Engineering, vol. 5, no. 3, 2016.

[41] E. F. E. Mehmet Onder, "Fractional order sliding mode control with reaching law approach," Turkish Journal of Electrical Engineering and Computer Sciences, vol. 18, no. 5, p. 731, 2010.

[42] D. Baleanu, K. Diethelm, E. Scalas, and J. J. Trujillo, Fractional Calculus: Models and Numerical Methods, World Scientific, Singapore, 2014.

[43] Y.Zhou, Basic Theory of Fractional Differential Equations, World Scientific, Singapore, 2014.

[44] K. Diethelm, The Analysis of Fractional Differential Equations, vol. 2004 of Lecture Notes in Mathematics, Springer, Berlin, Germany, 2010.

[45] H. K. Khalil, Nonlinear Systems, Prentice Hall, New York, NY, USA, 2002

[46] J. Slotine and W. Li, Applied Nonlinear Control, Prentice Hall, Upper Saddle River, NJ, USA, 1991.

[47] Z. Chen, X. Yuan, B. Ji, P. Wang, and H. Tian, "Design of a fractional order PID controller for hydraulic turbine regulating system using chaotic non-dominated sorting genetic algorithm II," Energy Conversion and Management, vol. 84, pp. 390-404, 2014.

[48] A. Ahuja and S. K. Aggarwal, "Design of fractional order pid controller for DC motor using evolutionary optimization techniques," WSEAS Transactions on Systems and Control, vol. 9, pp. 171-182, 2014.

[49] A. Charef, "Analogue realisation of fractional-order integrator, differentiator and fractional PID $\mu$ controller," IEE ProceedingsControl Theory and Applications, vol. 153, no. 6, pp. 714-720, 2006.

[50] Y. Q. Chen, B. M. Vinagre, and I. Podlubny, "Continued fraction expansion approaches to discretizing fractional order derivatives-an expository review," Nonlinear Dynamics, vol. 38, no. 1-4, pp. 155-170, 2004.

[51] Q. Wang, S. Yu, C. Li et al., "Theoretical design and FPGA-based implementation of higher-dimensional digital chaotic systems," IEEE Transactions on Circuits and Systems. I. Regular Papers, vol. 63, no. 3, pp. 401-412, 2016.

[52] X. Jiang, A systematic approach for digital hardware realization of fractional-order operators and systems [Ph.D. thesis], 2013, Ohiolink Database Accession Number: 1386649994.

[53] E. Tlelo-Cuautle, J. Rangel-Magdaleno, and L. G. de la Fraga, Engineering Applications of FPGAs_Chaotic Systems, Artificial Neural Networks, Random Number Generators, and Secure Communication Systems, Springer, 2016.

[54] C. Wang, R. Chu, and J. Ma, "Controlling a chaotic resonator by means of dynamic track control," Complexity, vol. 21, no. 1, pp. 370-378, 2015. 


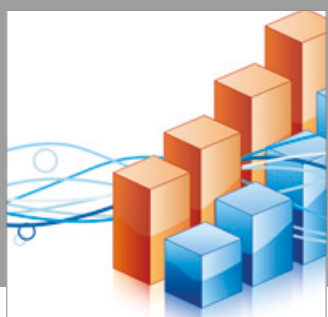

Advances in

Operations Research

vatersals

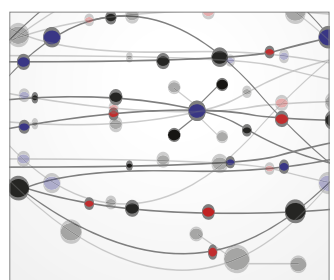

\section{The Scientific} World Journal
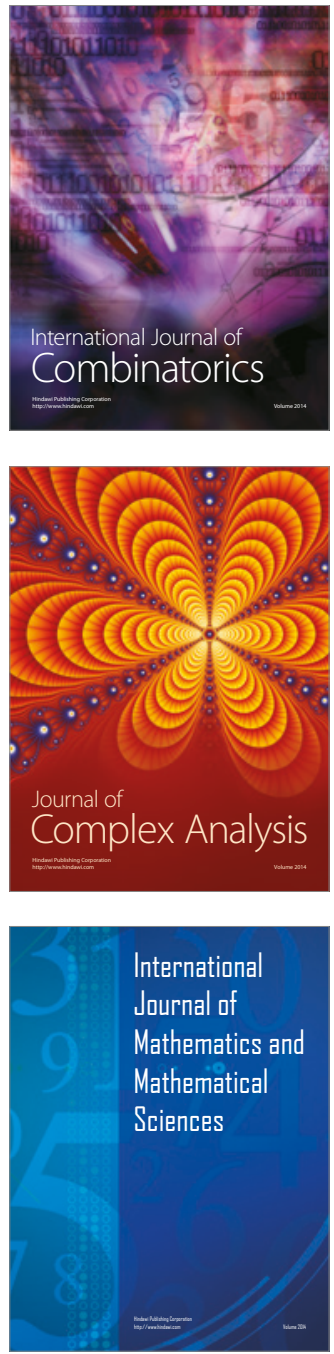
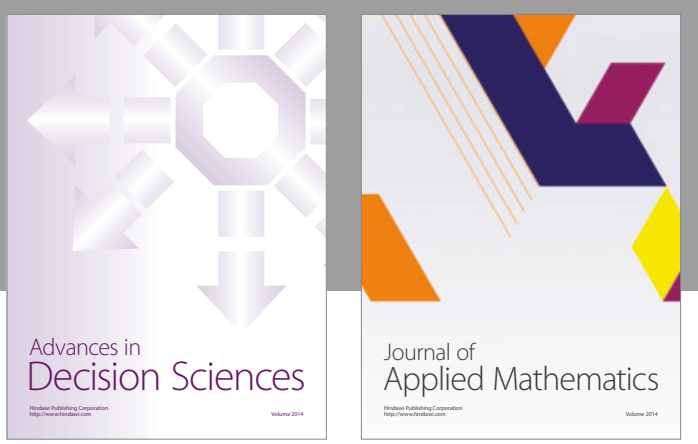

Algebra

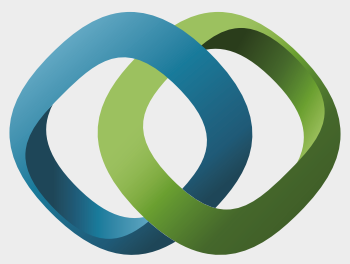

\section{Hindawi}

Submit your manuscripts at

https://www.hindawi.com
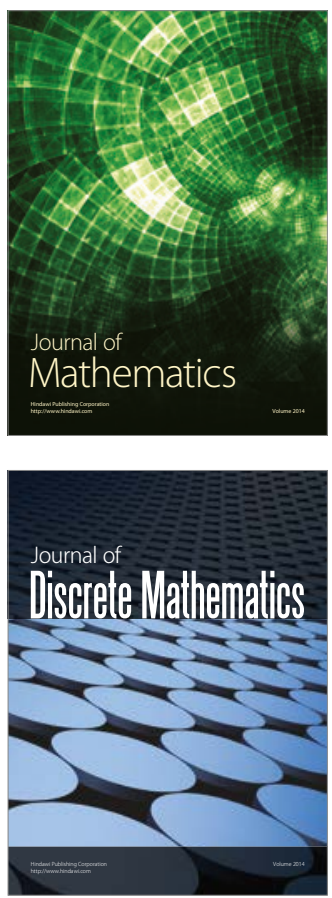

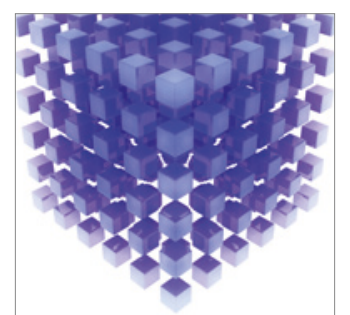

Mathematical Problems in Engineering
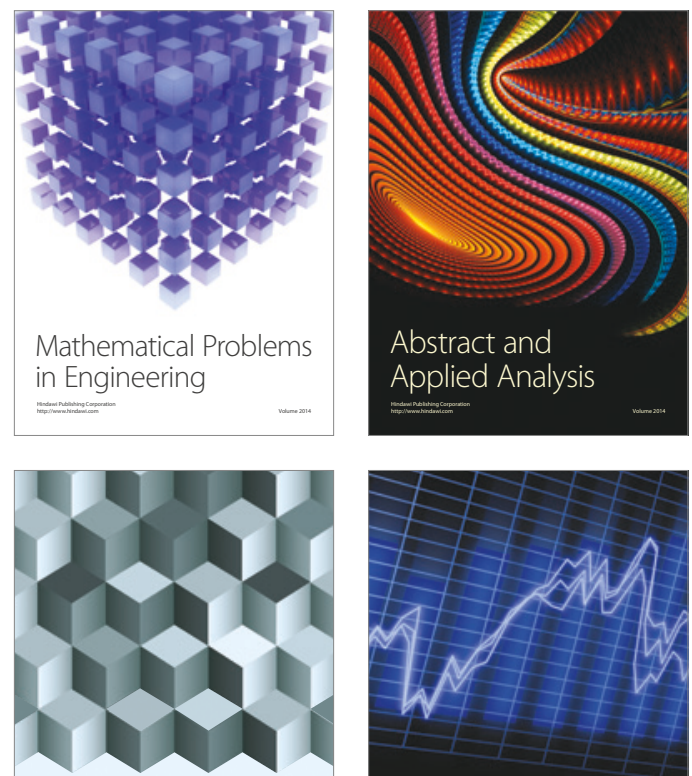

Journal of

Function Spaces

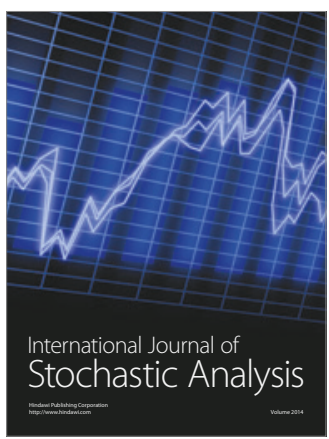

Probability and Statistics
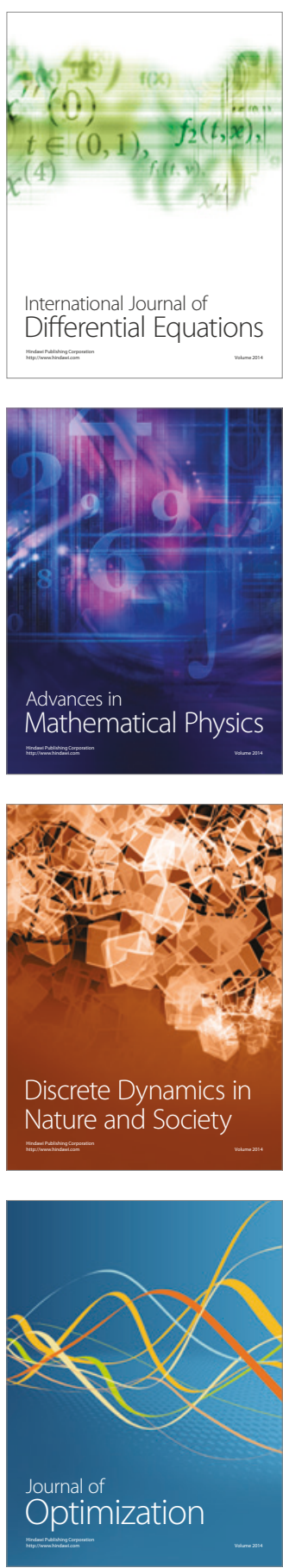